\title{
On the Abstract Inverse Scattering Problem for Trace Class Perturbations
}

\author{
R. Hatamleh \\ Department of Mathematics, Jadara University, Irbid-Jordan \\ E-mail: raedhat@yahoo.com \\ V.A. Zolotarev \\ B. Verkin Institute for Low Temperature Physics and Engineering \\ National Academy of Sciences of Ukraine \\ 47 Nauky Ave., Kharkiv 61103, Ukraine \\ E-mail: vazolotarev@gmail.com
}

Received July 15, 2015, revised November 3, 2015

The scattering problem for a pair of selfadjoint operators $\left\{L_{0}, L\right\}$, where $L-L_{0}$ is of trace-class, is studied. The explicit form of the scattering matrix and its properties are defined. The equation for the inverse problem is obtained.

Key words: scattering matrix, Friedrichs-Faddeev model, inverse scattering problem.

Mathematics Subject Classification 2010: 47A45.

\section{Introduction}

The scattering theory is not only the source of new non-traditional mathematical methods in the realm of perturbations of linear operators but it is also an important instrument for solving some problems of mathematical physics and performing calculus $[1,2]$. Along with the problems of scattering theory, such as the existence of wave operators and their completeness, the properties of scattering matrix etc. (the Kato-Birman theory), the inverse problem, contained in the reconstruction of the initial operator system by the physically measured scattering data, plays an important, if not the main, role [1-6].

In this paper, the scattering problem for the pair of selfadjoint operators $\left\{L_{0}, L\right\}$ is solved under the assumption that $L-L_{0}$ is a trace-class operator. Similar problems were also studied in [8-10]. For simplicity sake (although this does not complicate significantly the considerations mentioned below), the operator 
$L_{0}$ is assumed to be bounded. The main goal of the paper is to construct the scattering operator $S$ for the given pair $\left\{L_{0}, L\right\}$ and solve the inverse problem. The paper consists of 5 sections, in the first of which the wave operators $W_{ \pm}$ and the scattering operator $S$ are calculated using the methods of [2]. In Section 2 , for the operator system $\left\{L_{0}, L\right\}$ under study, the Friedrichs-Faddeev model is constructed and the scattering matrix $S(x)$ is calculated. The main properties of $S(x)$ are proven and the link between $S(x)$ and the function of spectral shift $\xi(x)$ is determined (the Birman-Krein Theorem). Section 3 is dedicated to the study of the Friedrichs-Faddeev model $\{\mathcal{Q}, \mathcal{L}\}$. The eigenfunctions of the operator $\mathcal{L}$ are described and the type of spectral projectors of absolutely continuous part of the spectrum of the operator $\mathcal{L}$ is defined. In Section 4, the main integral equation of the inverse problem (an analogue of the Marchenko equation) is obtained. The last section is dedicated to the solution of the inverse Riemann scattering problem for the case where $\operatorname{dim} E=1$. Similar problems for one-dimensional perturbations are studied in [19-21]. Notice that the statement of the problem is not traditional: the Friedrichs-Faddeev model is unambiguously restored by the scattering data. The solution of the inverse problem for the case $\operatorname{dim} E>1$ will be the subject of further publications.

\section{Scattering Operator}

I. In a separable Hilbert space $H$ we consider a pair of bounded linear selfadjoint operators $\left\{L_{0}, L\right\}$ such that

$$
L \stackrel{\text { def }}{=} L_{0}-\varphi^{*} R \varphi
$$

where $\varphi: H \rightarrow E$ ( $E$ is a separable Hilbert space); $R=R^{*}$ is an operator in $E$. It follows from (1) that

$$
R_{0}(\lambda)=R(\lambda)-R_{0}(\lambda) \varphi^{*} R \varphi R(\lambda) \quad\left(\lambda \notin \sigma\left(L_{0}\right) \cup \sigma(L)\right),
$$

where $R_{0}(\lambda)=\left(L_{0}-\lambda I\right)^{-1}$ and $R(\lambda)=(L-\lambda I)^{-1}$, and thus

$$
\varphi R(\lambda)=(I-V(\lambda) R)^{-1} \varphi R_{0}(\lambda),
$$

where $V(\lambda)$ is a Nevanlinna [9] operator-function in $E$,

$$
V(\lambda) \stackrel{\text { def }}{=} \varphi R_{0}(\lambda) \varphi^{*}
$$

Thus

$$
R(\lambda)=R_{0}(\lambda)+R_{0}(\lambda) \varphi^{*} N(\lambda) \varphi R_{0}(\lambda),
$$

where

$$
N(\lambda) \stackrel{\text { def }}{=} R(I-V(\lambda) R)^{-1}
$$


Theorem 1. The function $N(\lambda)$ (4) is Nevanlinna [12], $(N(\lambda)-R)$ is holomorphic at the infinitely distant point and the following statements are true: 1) $N(\lambda)=(I-R V(\lambda))^{-1} R$

2) $N^{*}(\lambda)=N(\bar{\lambda})$

3) $N(\lambda)-N(w)=N(\lambda)[V(\lambda)-V(w)] N(w)$

$=(\lambda-w) N(\lambda) \varphi R_{0}(\lambda) R_{0}(w) \varphi^{*} N(w)$;

4) the representation

$$
N(\lambda)=R+\int_{a}^{b} \frac{d \sigma(t)}{t-\lambda}
$$

is true, where $\lambda, w$ belong to the analyticity domain of $N(\lambda) ; V(\lambda)$ is given by $(2) ; R_{0}(\lambda)=\left(L_{0}-\lambda I\right)^{-1} ; \sigma(t)$ is a nondecreasing operator-function of bounded variation in $E ;-\infty<a<b<\infty$.

P r o o f. Relation 1) follows from the identity $(I-R V(\lambda)) R=R(I-V(\lambda) R)$. Equality 2) follows from 1) and from the fact that $R=R^{*}, V^{*}(\lambda)=V(\bar{\lambda})$. Since

$$
\begin{gathered}
N(\lambda)-N(w)=(I-R V(\lambda))^{-1} R[V(\lambda)-V(w)] R(I-V(w) R)^{-1} \\
=N(\lambda)[V(\lambda)-V(w)] N(w),
\end{gathered}
$$

$3)$ is a corollary of $V(\lambda)-V(w)=(\lambda-w) \varphi R_{0}(\lambda) R_{0}(w) \varphi^{*}$. Taking into account that

$$
N(\lambda)=R+R V(\lambda) R(I-V(\lambda) R)^{-1}, \quad \sup _{y>1}\|y V(i y)\|<\infty,
$$

we obtain 4) in view of the well-known [12] representation for the Nevanlinna functions.

For a selfadjoint operator $L$ in $H$ and for all $\varepsilon>0$,

$$
\int_{\mathbb{R}_{+}} e^{i \lambda t} e^{-i t L} e^{-\varepsilon t} d t=-i R(\lambda+i \varepsilon), \int_{\mathbb{R}_{+}} e^{-i \lambda t} e^{i t L} e^{-\varepsilon t} d t=i R(\lambda-i \varepsilon)
$$

take place, where $\lambda \in \mathbb{R}$ and $R(\lambda)=(L-\lambda I)^{-1}$. Consider an operator-function

$$
\delta(\lambda, \varepsilon) \stackrel{\text { def }}{=} \frac{1}{2 \pi i}\{R(\lambda+i \varepsilon)-R(\lambda-i \varepsilon)\}=\frac{\varepsilon}{\pi} R(\lambda+i \varepsilon) R(\lambda-i \varepsilon)
$$

$(\lambda \in \mathbb{R}, \varepsilon>0)$, then [2]

$$
\int_{\mathbb{R}} \delta(\lambda, \varepsilon) d \lambda=I
$$


II. We define the wave operators $[1-3]$,

$$
W_{ \pm} \stackrel{\text { def }}{=} s-\lim _{t \rightarrow \mp \infty} \exp \{i t L\} \cdot \exp \left\{-i t L_{0}\right\} P_{0}^{a},
$$

where $P_{0}^{a}$ is an orthoprojector on an absolutely continuous subspace of the operator $L_{0}$. The Kato-Rosenblum Theorem [1,2] yields that if $R$ is of trace-class, then the limits (9) exist and $W_{ \pm}$are complete, i. e., $W_{ \pm} H=P^{a} H$, where $P^{a}$ is an orthoprojector on an absolutely continuous subspace of the operator $L$. The operators $W_{ \pm}(9)$ are partial isometries from $P_{0}^{a} H$ on $P^{a} H[1,2]$ and they intertwine the absolutely continuous parts of the operators $L_{0}$ and $L$,

$$
L^{a} W_{ \pm}=W_{ \pm} L_{0}^{a}
$$

where $L_{0}^{a}=L_{0} P_{0}^{a}, L^{a}=L P^{a}$. The existence of $W_{ \pm}(9)$ yields that the limits $W_{ \pm}$exist in the weak convergence sense and these limits coincide. Use the weak convergence by Abel [2].

Theorem 2. If the limits $W_{ \pm}(9)$ exist, then

$$
\left\langle W_{ \pm} f_{0}, f\right\rangle=\lim _{\varepsilon \rightarrow+0} \int_{\mathbb{R}_{+}} 2 \varepsilon e^{-2 \varepsilon t}\left\langle\exp \{\mp i t L\} \exp \left\{ \pm i t L_{0}\right\} f_{0}, f\right\rangle d t
$$

$\forall f_{0} \in P_{0}^{a} H, \forall f \in P^{a} H$.

P r o o f. Let us show that $A \rightarrow 0$ as $\varepsilon \rightarrow+0$, where

$$
\begin{gathered}
A \stackrel{\text { def }}{=}\left\langle W_{-} f_{0}, f\right\rangle-2 \varepsilon \int_{\mathbb{R}_{+}} e^{-2 \varepsilon t}\left\langle e^{i t L} e^{-i t L_{0}} f_{0}, f\right\rangle d t= \\
=2 \varepsilon \int_{\mathbb{R}_{+}} e^{-2 \varepsilon t}\left\langle\left(W_{-}-e^{i t L} e^{-i t L_{0}}\right) f_{0}, f\right\rangle d t .
\end{gathered}
$$

For all $\delta>0$ there exists $N=N\left(\delta, f_{0}, f\right)$ such that $\left|\left\langle\left(W_{-} e^{i t L} e^{-i t L_{0}}\right) f_{0}, f\right\rangle\right|<\delta$ takes place for $t>N$, therefore

$$
\left|2 \varepsilon \int_{N}^{\infty} e^{-2 \varepsilon t}\left\langle\left(W_{-}-e^{i t L} e^{-i t L_{0}}\right) f_{0}, f\right\rangle d t\right|<\delta e^{-2 \varepsilon N} .
$$

Taking into account the unitarity of $e^{i t L}, e^{i t L_{0}}$ and the isometricity of $W_{-}$, we obtain

$$
\left|2 \varepsilon \int_{0}^{N} e^{-2 \varepsilon t}\left\langle\left(W_{-}-e^{i t L} e^{-i t L_{0}}\right) f_{0}, f\right\rangle d t\right| \leq 2\left\|f_{0}\right\| \cdot\|f\|\left(1-e^{-2 \varepsilon N}\right) .
$$


Using the obvious inequality $1-e^{-\delta} \leq \delta$, which is true for all $\delta \in \mathbb{R}_{+}$, we have $|A|<\delta e^{-2 \varepsilon N}+4 \varepsilon N\left\|f_{0}\right\| \cdot\|f\|$. Let $\varepsilon$ be such that $\varepsilon N<\delta$. Then $|A|<C \delta$ $\left(C=\max \left\{1,4\left\|f_{0}\right\| \cdot\|f\|\right\}\right)$.

Theorem 3. Let $R$ in (1) be of trace-class, then for the wave operators $W_{ \pm}$ (9), the following representations are true:

$$
\left\langle W_{ \pm} f_{0}, f\right\rangle=\left\langle f_{0}, f\right\rangle+\lim _{\varepsilon \rightarrow+0} \int_{\mathbb{R}}\left\langle R_{0}(\lambda \pm i \varepsilon) \varphi^{*} N(\lambda \pm i \varepsilon) \varphi \delta_{0}(\lambda, \varepsilon) f_{0}, f\right\rangle d t
$$

where $f_{0} \in P_{0}^{a} H, f \in P^{a} H$; the functions $N(\lambda)$ and $\delta(\lambda, \varepsilon)$ are given by (4) and (7), respectively; $R_{0}(\lambda)=\left(L_{0}-\lambda I\right)^{-1}$.

P r o o f. Since

$$
\left\langle W_{-} f_{0}, f\right\rangle=\lim _{\varepsilon \rightarrow+0} \int e_{\mathbb{R}_{+}} e^{-2 \varepsilon t}\left\langle e^{-i t L_{0}} f_{0}, e^{-i t L} f\right\rangle d t
$$

in view of (11), then using the Parseval inequality for the Fourier transform and (6), we obtain

$$
\left\langle W_{-} f_{0}, f\right\rangle=\lim _{\varepsilon \rightarrow+0} \frac{\varepsilon}{\pi} \int_{\mathbb{R}}\left\langle R_{0}(\lambda+i \varepsilon) f_{0}, R(\lambda+i \varepsilon) f\right\rangle d \lambda .
$$

Taking into account (3) and (7), we have

$$
\begin{gathered}
\left\langle W_{-} f_{0}, f\right\rangle= \\
=\lim _{\varepsilon \rightarrow+0}\left\{\int_{\mathbb{R}}\left\langle\delta_{0}(\lambda, \varepsilon) f_{0}, f\right\rangle d \lambda+\int_{\mathbb{R}}\left\langle R_{0}(\lambda-i \varepsilon) \varphi^{*} N(\lambda-i \varepsilon) \varphi \delta_{0}(\lambda, \varepsilon) f_{0}, f\right\rangle d \lambda\right\},
\end{gathered}
$$

which, in view of (8), gives us (12). For $W_{+}$, formula (12) is proved in a similar way.

Formula (12) implies

$$
\left\langle W_{\mp}^{*} f, f_{0}\right\rangle=\left\langle f, f_{0}\right\rangle+\lim _{\varepsilon \rightarrow+0} \int_{\mathbb{R}}\left\langle\delta_{0}(\mu, \varepsilon) \varphi^{*} N(\mu \pm i \varepsilon) \varphi R_{0}(\mu \pm i \varepsilon) f, f_{0}\right\rangle d \mu .
$$

Define the scattering operator [1-3],

$$
S \stackrel{\text { def }}{=} W_{+}^{*} W_{-} .
$$


The operator $S$ maps $P_{0}^{a} H$ onto itself isometrically, and

$$
S L_{0}^{a}=L_{0}^{a} S
$$

in view of (10), where $L_{0}^{a}$ is an absolutely continuous part of $L_{0}$.

Theorem 4. The scattering operator $S$ (14) of the pair $\left\{L_{0}, L\right\}$ ( $L$ is given by (1), $R$ is of trace-class) is given by the formula

$$
\left\langle(S-I) f_{0}, g_{0}\right\rangle=-2 \pi i \lim _{\varepsilon \rightarrow+0} \int_{\mathbb{R}}\left\langle\delta_{0}(\lambda, \varepsilon) \varphi^{*} N(\lambda-i \varepsilon) \varphi \delta_{0}(\lambda, \varepsilon) f_{0}, g_{0}\right\rangle d \lambda,
$$

where $f_{0}, g_{0} \in P_{0}^{a} H ; N(\lambda)$ and $\delta_{0}(\lambda, \varepsilon)$ are given by (4) and (7), respectively.

$$
\begin{aligned}
& \text { P r o o f. Formulas (12), (13) imply } \\
& \begin{array}{l}
\left\langle S f_{0}, g_{0}\right\rangle=\left\langle W_{-} f_{0}, W_{+} g_{0}\right\rangle=\left\langle f_{0}, W_{+} g_{0}\right\rangle+\lim _{\varepsilon \rightarrow+0} \int_{\mathbb{R}}\left\langle R_{0}(\lambda-i \varepsilon) \varphi^{*} N(\lambda-i \varepsilon) \varphi\right. \\
\left.\quad \times \delta_{0}(\lambda, \varepsilon) f_{0}, W_{+} g_{0}\right\rangle d \lambda=\left\langle f_{0}, g_{0}\right\rangle+\lim _{\varepsilon_{1} \rightarrow+0} \int_{\mathbb{R}}\left\langle\delta_{0}\left(\mu, \varepsilon_{1}\right) \varphi^{*} N\left(\mu-i \varepsilon_{1}\right) \varphi\right. \\
\left.\times R_{0}\left(\mu-i \varepsilon_{1}\right) f_{0}, g_{0}\right\rangle d \mu+\lim _{\varepsilon \rightarrow+0} \int_{\mathbb{R}}\left\langle R_{0}(\lambda-i \varepsilon) \varphi^{*} N(\lambda-i \varepsilon) \varphi \delta_{0}(\lambda, \varepsilon) f_{0}, g_{0}\right\rangle d \lambda \\
+\lim _{\varepsilon_{1} \rightarrow+0} \lim _{\varepsilon \rightarrow+0} \int_{\mathbb{R}} d \lambda \int_{\mathbb{R}} d \mu\left\langle\delta_{0}\left(\mu, \varepsilon_{1}\right) \varphi^{*} N\left(\mu-i \varepsilon_{1}\right) \varphi R_{0}\left(\mu-i \varepsilon_{1}\right) R_{0}(\lambda-i \varepsilon) \varphi^{*}\right. \\
\left.\quad \times N(\lambda-i \varepsilon) \varphi \delta_{0}(\lambda, \varepsilon) f_{0}, g_{0}\right\rangle .
\end{array}
\end{aligned}
$$

The double integral, in view of 3 ) (5), is equal to

$$
B \stackrel{\text { def }}{=} \int_{\mathbb{R}} d \lambda \int_{\mathbb{R}} d \mu\left\langle\delta_{0}\left(\mu, \varepsilon_{1}\right) \varphi^{*} \frac{N\left(\mu-i \varepsilon_{1}\right)-N(\lambda-i \varepsilon)}{\mu-\lambda+i\left(\varepsilon-\varepsilon_{1}\right)} \varphi \delta_{0}(\lambda, \varepsilon) f_{0}, g_{0}\right\rangle .
$$

Calculate the expression

$$
\begin{gathered}
\int_{\mathbb{R}} \frac{d \lambda}{\mu-\lambda+i\left(\varepsilon-\varepsilon_{1}\right)} \delta_{0}(\lambda, \varepsilon)=\frac{1}{2 \pi i} \int_{\mathbb{R}} \frac{d \lambda}{\mu-\lambda+i\left(\varepsilon_{1}-\varepsilon\right)}\left\{\int_{\mathbb{R}} \frac{d E_{x}}{x-\lambda-i \varepsilon}\right. \\
\left.-\int_{\mathbb{R}} \frac{d E_{x}}{x-\lambda+i \varepsilon}\right\}=\frac{1}{2 \pi i} \int_{\mathbb{R}} \frac{d E_{x}}{x-\mu+i\left(\varepsilon_{1}-2 \varepsilon\right)}\left\{\int _ { \mathbb { R } } \left[\frac{1}{\lambda-x+i \varepsilon}\right.\right.
\end{gathered}
$$




$$
\begin{gathered}
\left.\left.-\frac{1}{\left.\lambda-\mu+i\left(\varepsilon_{1}-\varepsilon\right)\right)}\right] d \lambda\right\}-\frac{1}{2 \pi i} \int_{\mathbb{R}} \frac{d E_{x}}{x-\mu+i \varepsilon_{1}}\left\{\int _ { \mathbb { R } } \left[\frac{1}{\lambda-x-i \varepsilon}\right.\right. \\
\left.\left.-\frac{1}{\mu-\lambda+i\left(\varepsilon_{1}-\varepsilon\right)}\right] d \lambda\right\} .
\end{gathered}
$$

If $\varepsilon>\varepsilon_{1}$, then

$$
\begin{gathered}
\int_{\mathbb{R}}\left[\frac{1}{\lambda-x+i \varepsilon}-\frac{1}{\lambda-\mu+i\left(\varepsilon_{1}-\varepsilon\right)}\right] d \lambda=-2 \pi i, \\
\int_{\mathbb{R}}\left[\frac{1}{\lambda-x-i \varepsilon}-\frac{1}{\mu-\lambda+i\left(\varepsilon_{1}-\varepsilon\right)}\right] d \lambda=0,
\end{gathered}
$$

and

$$
\int_{\mathbb{R}} \frac{d \lambda}{\mu-\lambda+i\left(\varepsilon-\varepsilon_{1}\right)} \delta_{0}(\mu, \varepsilon)=-R_{0}\left(\mu-i\left(2 \varepsilon-\varepsilon_{1}\right)\right) \quad\left(\varepsilon>\varepsilon_{1}\right) .
$$

Analogously,

$$
\int_{\mathbb{R}} \frac{d \mu}{\mu-\lambda+i\left(\varepsilon-\varepsilon_{1}\right)} \delta_{0}\left(\mu, \varepsilon_{1}\right)=R_{0}\left(\lambda_{1}-\varepsilon\right) \quad\left(\varepsilon>\varepsilon_{1}\right) .
$$

Calculate the double integral

$$
\begin{gathered}
B=-\int_{\mathbb{R}} d \mu\left\langle\delta_{0}\left(\mu, \varepsilon_{1}\right) \varphi^{*} R_{0}\left(\mu-i\left(2 \varepsilon-\varepsilon_{1}\right)\right) f_{0}, g_{0}\right\rangle \\
-\int_{\mathbb{R}} d \lambda\left\langle R_{0}(\lambda-i \varepsilon) \varphi^{*} N(\lambda-i \varepsilon) \varphi \delta_{0}(\lambda, \varepsilon) f_{0}, g_{0}\right\rangle .
\end{gathered}
$$

Substituting this expression into the sum for $\left\langle S f_{0}, g_{0}\right\rangle$, we obtain

$$
\begin{gathered}
\left\langle(S-I) f_{0}, g_{0}\right\rangle=\lim _{\varepsilon, \varepsilon_{1} \rightarrow+0} \int_{\mathbb{R}} d \mu\left\langle\delta\left(\mu, \varepsilon_{1}\right) \varphi^{*} N\left(\mu-i \varepsilon_{1}\right) \varphi \times\right. \\
\left.\quad \times\left\{R_{0}\left(\mu-i \varepsilon_{1}\right)-R_{0}\left(\mu+i\left(2 \varepsilon-\varepsilon_{1}\right)\right)\right\} f_{0}, g_{0}\right\rangle .
\end{gathered}
$$

Proceeding to the limit as $\varepsilon \rightarrow \varepsilon_{1}+0\left(\varepsilon>\varepsilon_{1}\right)$ and taking into account (7), we obtain (16). For $\varepsilon_{1}>\varepsilon$, the considerations are similar and we again obtain formula (16). 


\section{The Friedrichs-Faddeev Model}

III. Consider the subspace $H_{1}(\subseteq H)$,

$$
H_{1} \stackrel{\text { def }}{=} \operatorname{span}\left\{R_{0}(\lambda) \varphi^{*} E: \lambda \in \mathbb{C} \backslash \sigma\left(L_{0}\right)\right\},
$$

where $R_{0}(\lambda)=\left(L_{0}-\lambda I\right)^{-1}$. The subspace $H_{1}$ reduces the operators $L_{0}$ and $L_{1}$ (1). Since $H_{1}^{\perp} \subset \operatorname{Ker} \varphi$, then $\left.L\right|_{H_{1}^{\perp}}=\left.L_{0}\right|_{H_{0}^{\perp}}$ and the wave operators $W_{ \pm}$(9) corresponding to these restrictions coincide with the projector $P_{1}^{a}\left(P_{1}^{a}\right.$ is a projector on the absolutely continuous subspace of $\left.\left.L_{0}\right|_{H_{1}^{\perp}}\right)$. The scattering operator $S$ (14) on $H_{1}^{\perp}$ is a trivial one; $\left.S\right|_{H_{1}^{\perp}}=P_{1}^{a}$. Therefore the supposition that $H=H_{1}$ (17) is reasonable, and thus $\overline{\varphi^{*} E}$ is the generating subspace of the operator $L_{0}$ [13].

In $E$, specify a non-descending operator-function, $F(x)=\varphi E_{x} \varphi^{*}$, where $E_{x}$ is the resolution of identity of the operator $L_{0}$. The operator $L_{0}$ being bounded, the support of the measure $d F(x)$ is finite. Define the Hilbert space

$$
L_{\mathbb{R}}^{2}(E, d F(x)) \stackrel{\text { def }}{=}\left\{f(x) \in E: \int_{\mathbb{R}}\langle d F(x) f(x), f(x)\rangle<\infty\right\}
$$

obtained as a result of the closure of the class of continuous vector-functions $f(x)$ from $E$. If $H_{1}=H$, then the operator $U: L_{\mathbb{R}}^{2}(E, d F(x)) \rightarrow H$, given by the formula

$$
f=U f(x), \quad f \stackrel{\text { def }}{=} \int_{\mathbb{R}} d E_{x} \varphi^{*} f(x)
$$

$\left(f \in H, f(x) \in L_{\mathbb{R}}^{2}(E, d F(x))\right)$, specifies the unitary isomorphism between $H$ $\left(=H_{1}\right)$ and $L_{\mathbb{R}}^{2}(E, d F(x))(18)$.

Theorem 5. Let $H=H_{1}(17)$ and $U: L_{\mathbb{R}}^{2}(E, d F(x)) \rightarrow H$ be given by (19), then the operators $\mathcal{Q}=U^{*} L_{0} U$ and $\mathcal{L}=U^{*} L U$ are

$$
(\mathcal{Q} f)(x) \stackrel{\text { def }}{=} x f(x), \quad(\mathcal{L} f) \stackrel{\text { def }}{=} x f(x)-R \int_{\mathbb{R}} d F(t) f(t),
$$

where $f(x) \in L_{\mathbb{R}}^{2}(E, d F(x))$.

P r o o f. The representation of $\mathcal{Q}$ (20) follows from the equality

$$
L_{0} U f(x)=L_{0} \int_{\mathbb{R}} d E_{x} \varphi^{*} f(x)=\int_{\mathbb{R}} d E_{x} \varphi^{*}(x f(x))=U \mathcal{Q} f(x) .
$$

Since $\tilde{\varphi}=\varphi U$ is given by

$$
\tilde{\varphi} f(x)=\int_{\mathbb{R}} d F(x) f(x)
$$


then, taking into account that $\tilde{\varphi}^{*}$ is the operator of enclosure of $E$ into $L_{\mathbb{R}}^{2}(E, d F(x))$, we obtain the representation of $\mathcal{L}$ in view of (1).

Formulas (20), well-known in the scattering theory [2, 4], are called the Friedrichs-Faddeev model.

The scattering operator $S(14)$ corresponds to the operator of multiplication by the operator-function $S(x)=U^{*} S U$ in $E$ ( $U$ is given by (19)), which is commonly $[1,2]$ named a scattering matrix. The above follows from the commutativity of $U^{*} S U$ and $\mathcal{Q}(20)$ in view of (15).

Further, we will suppose that the operator $\varphi$ from (1) belongs to the HilbertShmidt class. Denote the function $F(x)=\varphi E_{x} \varphi^{*}$ which also belongs to this class for all $x \in \mathbb{R}$. Moreover, we suppose that the function $F(x)$ is absolutely continuous in the sense that there exists a nonnegative integrable (by Bochner) operator-function $a(\cdot): \mathbb{R} \rightarrow B(E),(B(E)$ is the space of bounded operators in $E)$, such that

$$
F(\Delta)=\int_{\Delta} a(x) d x \quad(a(x) \geq 0)
$$

for any Borel set $\Delta$ from $\mathbb{R}$. Formally, we write this condition of absolute continuity in the form

$$
d F(x)=a(x) d x .
$$

Theorem 6. Let $H=H_{1}(17)$, and the function $F(x)=\varphi E_{x} \varphi^{*}$ be absolutely continuous, moreover, we suppose that $R$ in (1) is of trace-class and $\varphi$ belongs to the Hilbert-Schmidt class. Then the scattering matrix $S(x)=U^{*} S U$ corresponding to $S$ (14) is given by

$$
S(x)=I-2 \pi i N_{-}(x) a(x) \quad(x \in \mathbb{R}),
$$

where $N_{-}(x)=N(x-i 0)$ are the boundary values on $\mathbb{R}$ from $\mathbb{C}_{-}$of the function $N(\lambda)(4)$.

P r o o f. Let

$$
f_{0}=\int_{\mathbb{R}} d E_{x} \varphi^{*} f_{0}(x) \quad\left(f_{0} \in L_{\mathbb{R}}^{2}(E, d F(x))\right) .
$$

Then (16) implies

$$
\begin{gathered}
\left\langle(S-I) f_{0}, g_{0}\right\rangle \\
=-2 \pi i \lim _{\varepsilon \rightarrow+0} \int_{\mathbb{R}}\left\langle\delta_{0}(\lambda, \varepsilon) \varphi^{*} N(\lambda-i \varepsilon) \varphi \delta_{0}(\lambda, \varepsilon) \int_{\mathbb{R}} d E_{x} \varphi^{*} f_{0}(x), g_{0}\right\rangle d \lambda .
\end{gathered}
$$


Since

$$
\begin{gathered}
\varphi \delta_{0}(\lambda, \varepsilon) \int_{\mathbb{R}} d E_{x} \varphi^{*} f_{0}(x)=\frac{\varepsilon}{\pi} \varphi \int_{\mathbb{R}} d E_{x} \varphi^{*} f_{0}(x) \\
=\frac{\varepsilon}{\pi} \int_{\mathbb{R}} \frac{d F(x) f_{0}(x)}{(x-\lambda)^{2}+\varepsilon^{2}}=\frac{1}{\pi} \int_{\mathbb{R}} \frac{\varepsilon}{(x-\lambda)^{2}+\varepsilon^{2}} a(x) f_{0}(x) d x,
\end{gathered}
$$

then, using the properties of the Poisson kernel [2, 14, 15], we obtain

$$
\varphi \delta_{0}(\lambda, x) \int_{\mathbb{R}} d E_{x} \varphi^{*} f_{0}(x) \rightarrow a(\lambda) f_{0}(\lambda) \quad(\varepsilon \rightarrow+0) .
$$

Taking into account [13, 14],

$$
\left\langle\delta_{0}(\lambda, \varepsilon) f, g\right\rangle \rightarrow \frac{d}{d \lambda}\left\langle E_{\lambda} f, g\right\rangle \quad(\varepsilon \rightarrow+0),
$$

and the existence of the boundary values $N(\lambda-i \varepsilon) \rightarrow N_{-}(\lambda)(=N(\lambda-i 0))$ as $\varepsilon \rightarrow+0[11,12]$, we obtain

$$
\left\langle(S-I) f_{0}, g_{0}\right\rangle=-2 \pi i \int_{\mathbb{R}}\left\langle d E_{\lambda} \varphi^{*} N_{-}(\lambda) a(\lambda) f_{0}(\lambda), g_{0}\right\rangle .
$$

Hence,

$$
S f_{0}=\int_{\mathbb{R}} d E_{\lambda} \varphi^{*}\left\{I-2 \pi i N_{-}(\lambda) a(\lambda)\right\} f_{0}(\lambda)=U\left\{I-2 \pi i N_{-}(\lambda) a(\lambda)\right\} f_{0}(\lambda),
$$

which gives us (22).

Let

$$
S^{+}=S^{*} \stackrel{\text { def }}{=} W_{-}^{*} W_{+}
$$

Then, similarly to (16),

$$
\left\langle\left(S^{+}-I\right) f_{0}, g_{0}\right\rangle=2 \pi i \lim _{\varepsilon \rightarrow+0} \int_{\mathbb{R}}\left\langle\delta_{0}(\lambda, \varepsilon) \varphi^{*} N(\lambda+i \varepsilon) \varphi \delta_{0}(\lambda, \varepsilon) f_{0}, g_{0}\right\rangle d \lambda,
$$

and thus the scattering matrix $S^{+}(x)=U^{*} S^{+} U$ can be written in the form

$$
S^{+}(x)=I+2 \pi i N_{+}(x) a(x) \quad(x \in \mathbb{R}),
$$

where $N_{+}(x)=N(x+i 0)$ are the boundary values on $\mathbb{R}$ from $\mathbb{C}_{+}$of the function $N(\lambda)(4)$. 
It is obvious that as $x \notin \overline{\operatorname{supp} a(x)}$ the matrices $S(x)(22)$ and $S^{+}(x)(25)$ are equal to the identity operator, moreover,

$$
a(x) S^{+}(x)=S^{*}(x) a(x) \quad(x \in \mathbb{R}) .
$$

Hence, $S^{+}(x)$ is adjoint to $S(x)$ in the metric of $L_{\mathbb{R}}^{2}(E, d F(x))$.

Let us find the functional realizations of the wave operators $W_{ \pm}(12)$. Substitute $f_{0}(23)$ into representation (12) for $W_{-}$, then

$$
\begin{gathered}
\left\langle\left(W_{-}-I\right) f_{0}, f\right\rangle \\
=\lim _{\varepsilon \rightarrow+0} \int_{\mathbb{R}}\left\langle R_{0}(\lambda-i \varepsilon) \varphi^{*} N(\lambda-i \varepsilon) \varphi \delta_{0}(\lambda, \varepsilon) \int_{\mathbb{R}} d E_{x} \varphi^{*} f_{0}(x), f\right\rangle d \lambda \\
=\lim _{\varepsilon \rightarrow+0} \int_{\mathbb{R}}\left\langle d E_{y} \varphi^{*} \int_{\mathbb{R}} \frac{N(\lambda-i \varepsilon)}{y-\lambda+i \varepsilon} \varphi \delta_{0}(\lambda, \varepsilon) \int_{\mathbb{R}} d E_{x} \varphi^{*} f_{0}(x), f\right\rangle d \lambda,
\end{gathered}
$$

which gives

$$
\left\langle\left(W_{-}-I\right) f_{0}, f\right\rangle=-\int_{\mathbb{R}}\left\langle d E_{x} \varphi^{*} \int_{\mathbb{R}} \frac{N_{-}(\lambda)}{\lambda-x-i 0} a(\lambda) f(\lambda) d \lambda, f\right\rangle .
$$

Theorem 7. If $H=H_{1}$ (17), the function $F(x)=\varphi E_{x} \varphi^{*}$ is absolutely continuous (21), the operator $R$ from (1) is of trace-class, and $\varphi$ is a HilbertSchmidt operator, then the wave operators $\widetilde{W}_{ \pm}=U^{*} W_{ \pm} U$ are

$$
\left(\widetilde{W}_{\mp} f\right)(x)=f(x)-\int_{\mathbb{R}} \frac{N_{\mp}(\lambda)}{\lambda-x \mp i 0} a(\lambda) f(\lambda) d \lambda,
$$

where $f(x) \in L_{\mathbb{R}}^{2}(E, d F(x))$.

Using (22), (24), we obtain

$$
\begin{aligned}
& \left(\widetilde{W}_{-} f\right)(x)=f(x)-\frac{1}{2 \pi i} \int_{\mathbb{R}} \frac{I-S(\lambda)}{\lambda-x-i 0} f(\lambda) d \lambda, \\
& \left(\widetilde{W}_{+} f\right)(x)=f(x)-\frac{1}{2 \pi i} \int_{\mathbb{R}} \frac{S^{+}(\lambda)-I}{\lambda-x+i 0} f(\lambda) d \lambda,
\end{aligned}
$$

which, by the Sokhotsky formulas [16], gives us the representations

$$
\begin{aligned}
& \left(\widetilde{W}_{-} f\right)(x)=\frac{1}{2}(I+S(\lambda)) f(\lambda)-\frac{1}{2 \pi i} \int_{\mathbb{R}} \frac{I-S(\lambda)}{\lambda-x} f(\lambda) d \lambda, \\
& \left(\widetilde{W}_{+} f\right)(x)=\frac{1}{2}\left(S^{+}(\lambda)+I\right) f(\lambda)-\frac{1}{2 \pi i} \int_{\mathbb{R}} \frac{S^{+}(\lambda)-I}{\lambda-x} d \lambda .
\end{aligned}
$$


The intertwining conditions follow directly from (10),

$$
\mathcal{L}^{a} \widetilde{W}_{ \pm}=\widetilde{W}_{ \pm} \mathcal{Q}^{a}
$$

Construct a direct proof. Using (27), we get

$$
\begin{aligned}
\left(\widetilde{W}_{-} \mathcal{Q}^{a} f\right)(x) & =x f(x)-\pi i N_{-}(x) a(x) x f(x)-\int_{\mathbb{R}} \frac{N_{-}(\lambda)}{\lambda-x} a(\lambda) \lambda f(\lambda) \\
& =x\left(\widetilde{W}_{-} f\right)(x)-\int_{\mathbb{R}} N_{-}(\lambda) a(\lambda) f(\lambda) d \lambda .
\end{aligned}
$$

Taking into account the identity $N(\lambda)=R+R V(\lambda) N(\lambda)$, we obtain

$$
\begin{gathered}
\left(\widetilde{W}_{-} \mathcal{Q}^{a} f\right)(x)=x\left(\widetilde{W}_{-} f\right)(x)-R \int_{\mathbb{R}} a(\lambda) f(\lambda)-R \int_{\mathbb{R}}\left\{-\pi i a(\lambda)+\int_{\mathbb{R}} \frac{a(t) d t}{t-\lambda}\right\} \\
\times N_{-}(\lambda) a(\lambda) f(\lambda) d \lambda=x\left(\widetilde{W}_{-} f\right)(x)-R \int_{\mathbb{R}} a(\lambda)\left\{f(\lambda)-\pi i N_{-}(\lambda) a(\lambda) f(\lambda)\right. \\
\left.-\int_{\mathbb{R}} \frac{N_{-}(x)}{x-\lambda} a(x) f(x) d x\right\} d \lambda=\mathcal{L}^{a}\left(\widetilde{W}_{-} f\right)(x) .
\end{gathered}
$$

Equalities (30) show that $\widetilde{W}_{ \pm}(27)$ are the transformation operators $[4,5]$.

IV. The absolute continuity of $F(x)(21)$ and (7) imply

$$
a(x)=\lim _{\varepsilon \rightarrow+0} \varphi \delta_{0}(\lambda, \varepsilon) \varphi=\frac{1}{2 \pi i} \lim _{\varepsilon \rightarrow+0}[V(x+i \varepsilon)-V(x-i \varepsilon)],
$$

thus

$$
V_{+}(x)-V_{-}(x)=2 \pi i a(x),
$$

where $V_{ \pm}(x)$ are the boundary values on $\mathbb{R}$ from $\mathbb{C}_{ \pm}$of the function $V(\lambda)(2)$. Equality (31) coincides with the Stieltjes-Perron inversion formula [2, 12] and may also follow from the Sokhotsky formula [16] for $V_{ \pm}(x)$.

Using 3) (5) and (31), we obtain

$$
\begin{aligned}
& N_{+}(x)-N_{-}(x)=2 \pi i N_{+}(x) a(x) N_{-}(x) \\
& N_{+}(x)-N_{-}(x)=2 \pi i N_{-}(x) a(x) N_{+}(x),
\end{aligned}
$$

where $N_{ \pm}(x)$ are the boundary values on $\mathbb{R}$ from $\mathbb{C}_{ \pm}$of the function $N(\lambda)(4)$. 
Theorem 8. If the representing measure $F(x)$ of the function $V(\lambda)$ (2) is absolutely continuous (21) and the suppositions of Theorem 6 take place, then

$$
\begin{array}{ll}
\text { 1) } & S^{*}(x) a(x) S(x)=a(x), \quad\left(S^{+}(x)\right)^{*} a(x) S^{+}(x)=a(x), \\
2) & S^{+}(x) S(x)=S(x) S^{+}(x)=I,
\end{array}
$$

where $S(x)$ and $S^{+}(x)$ are given by (22) and (25).

P r o o f. The definition of $S(x)$ from (22) implies

$$
\begin{gathered}
S^{*}(x) a(x) S(x)=a(x)-2 \pi i a(x) N_{-}(x) a(x)+2 \pi i a(x) N_{+}(x) a(x)- \\
-(2 \pi i)^{2} a(x) N_{+}(x) a(x) N_{-}(x) a(x)=a(x)
\end{gathered}
$$

in view of (32). We prove the second relation from 1) in a similar way. Taking into account (22), (25), and (32), we obtain

$S^{+}(x) S(x)=I-2 \pi i N_{-}(x) a(x)+2 \pi i N_{+}(x) a(x)-(2 \pi i)^{2} N_{+}(x) a(x) N_{-}(x) a(x)=I$

in view of (32).

Corollary 1. The equalities 1) (33) prove that the reciprocal matrix-functions $S(x)$ and $S^{+}(x)$ are unitary in the metric of the space $L_{\mathbb{R}}^{2}(E, d F(x))(18)$ when $d F(x)=a(x) d x$.

Using (22) and (31), we obtain

$$
S(x)=I-\left(I-R V_{-}(x)\right)^{-1} R\left(V_{+}(x)-V_{-}(x)\right)=\left(I-R V_{-}(x)\right)^{-1}\left(I-R V_{+}(x)\right) \text {, }
$$

therefore

$$
S(x)=\left(I-R V_{-}(x)\right)^{-1}\left(I-R V_{+}(x)\right) .
$$

Thus,

$$
S(x)\left(I-R V_{+}(x)\right)^{-1}=\left(I-R V_{-}(x)\right)^{-1},
$$

which, in view of 1) (5), gives us

$$
S(x) N_{+}(x)=N_{-}(x) .
$$

Theorem 9. Let the suppositions of Theorem 6 be true. Then the Nevanlinna function $N(\lambda)$ (4) is the solution of the Riemann boundary value problem [16], (35), the coefficient of which is the scattering matrix $S(x)(22)$.

The equalities 2) (33) are dual to (34),

$$
S^{+}(x) N_{-}(x)=N_{+}(x),
$$

where $S^{+}(x)$ is given by $(25)$. 
V. From (3) and the fact that $N(\lambda)$ (4) is of trace-class we have

$$
\operatorname{tr}\left\{R(\lambda)-R_{0}(\lambda)\right\}=\operatorname{tr}\left\{\varphi R_{0}^{2}(\lambda) \varphi^{*} N(\lambda)\right\}=\operatorname{tr}\left\{V^{\prime}(\lambda) N(\lambda)\right\} .
$$

Using (4) and the formula from [17],

$$
\frac{d}{d \lambda} \ln \operatorname{det}(I-A(\lambda))=-\operatorname{tr}\left\{A^{\prime}(\lambda)(I-A(\lambda))^{-1}\right\},
$$

we obtain

$$
\operatorname{tr}\left\{V^{\prime}(\lambda) N(\lambda)\right\}=-\frac{d}{d \lambda} D(\lambda)
$$

where

$$
D(\lambda) \stackrel{\text { def }}{=} \operatorname{det}(I-R V(\lambda))
$$

is the perturbation operator [17] of the pair of operators $\mathcal{Q}, \mathcal{L}(20)$. The existence of (37) is determined by the fact that $R$ is of trace-class.

The following statement belongs to M. G. Krein $[2,18]$.

Theorem 10. Let $R$ be of trace-class. Then for $D(\lambda)(37)$,

$$
\ln D(\lambda)=-\int_{\mathbb{R}} \frac{\xi(x)}{x-\lambda} d x \quad(\operatorname{Im} \lambda \neq 0)
$$

takes place, where $\xi(x)$ is the spectral shift function [2, 18],

$$
\xi(x) \stackrel{\text { def }}{=}-\frac{1}{\pi} \lim _{\varepsilon \rightarrow+0} \arg D(x+i \varepsilon) .
$$

The limit (38) exists almost everywhere for $x \in \mathbb{R}$, and

$$
\int_{\mathbb{R}}|\xi(x)| d x \leq\|R\|_{1} ; \quad \int_{\mathbb{R}} \xi(x) d x=\operatorname{tr} R
$$

$\left(\|R\|_{1}\right.$ is the trace-class norm of $\left.R\right)$. Moreover, $\xi(x) \leq k_{+}\left(\xi(x) \geq k_{-}\right)$almost everywhere if $R$ has exactly $k_{+}$positive ( $k_{-}$negative) eigenvalues.

Using this theorem, we obtain

$$
\operatorname{tr}\left\{R(\lambda)-R_{0}(\lambda)\right\}=\int_{\mathbb{R}} \frac{\xi(x)}{(x-\lambda)^{2}} d x \quad(\operatorname{Im} \lambda \neq 0),
$$

which implies the well-known Krein trace formula [2, 18].

Theorem 11 (M. G. Krein). Let $R$ be of trace-class and $f(\lambda)$ be a holomorphic function as $\lambda \in \sigma\left(L_{0}\right) \cup \sigma\left(L_{1}\right)$, then

$$
\operatorname{tr}\left\{f(L)-f\left(L_{0}\right)\right\}=\int_{\mathbb{R}} \xi(x) f^{\prime}(x) d x .
$$


The link between a scattering matrix and a function of spectral shift was established by M. Sh. Birman and M. G. Krein [2].

Theorem 12 (M. Sh. Birman, M. G. Krein). If $R$ is of trace-class, then for almost all $x \in \mathbb{R}$ the formula

$$
\operatorname{det} S(x)=\exp \{-2 \pi i \xi(x)\}
$$

is true, where $S(x)$ is the scattering matrix (22), and $\xi(x)$ is the function of spectral shift (39).

P r o o f. Equality (34) yields

$$
\operatorname{det} S(x)=D(x+i 0) D^{-1}(x-i 0),
$$

where $D(\lambda)$ is given by (37). To conclude the proof it remains to use definition (39) of the function $\xi(x)$.

\section{Scattering Matrix}

VI. We proceed to the analysis of the Friedrichs-Faddeev model (20) assuming that $F(x)$ is absolutely continuous $(21)$. To find the resolvent $R_{\mathcal{L}}(\lambda)=(\mathcal{L}-\lambda I)^{-1}$, we denote $(\mathcal{L}-\lambda I)^{-1} f(x)=g(x)$. Then

$$
(x-\lambda) g(x)-R \int_{\mathbb{R}} a(t) g(t) d t=f(x) .
$$

In terms of $G(x)=(x-\lambda) g(x)$ this equation takes the form

$$
G(x)-R \int_{\mathbb{R}} a(t) \frac{G(t)}{t-\lambda} d t=f(x) .
$$

Multiplying this equality by $a(x)(x-\lambda)^{-1}$ and integrating it, we obtain

$$
\int_{\mathbb{R}} a(x) \frac{G(x)}{x-\lambda} d x-V(\lambda) R \int_{\mathbb{R}} a(t) \frac{G(t)}{t-\lambda} d t=\int_{\mathbb{R}} a(x) \frac{f(x)}{x-\lambda} d x .
$$

Hence,

$$
\int_{\mathbb{R}} a(x) \frac{G(x)}{x-\lambda} d x=(I-V(\lambda) R)^{-1} \int_{\mathbb{R}} a(x) \frac{f(x)}{x-\lambda} d x .
$$


Theorem 13. The resolvent $R_{\mathcal{L}}(\lambda)=(\mathcal{L}-\lambda I)^{-1}$ of the operator $\mathcal{L}(20)$ is given by

$$
\left(R_{\mathcal{L}}(\lambda) f\right)(x)=\frac{f(x)}{x-\lambda}+\frac{N(\lambda)}{x-\lambda} \int_{\mathbb{R}} a(t) \frac{f(t)}{t-\lambda} d t,
$$

where $f(x) \in L_{\mathbb{R}}^{2}(E, d F(x))$, the function $F(x)$ is absolutely continuous (21), and $N(\lambda)$ is given by formula (4).

The description of the discrete spectrum $\sigma_{p}(\mathcal{L})$ of the operator $\mathcal{L}(20)$ lies in the following.

Theorem 14. Suppose that the function $F(x)=\varphi E_{x} \varphi^{*}$ is absolutely continuous and $R$ is of trace-class. Then the eigenfunctions $\mathcal{L} f_{\lambda}(x)=\lambda f_{\lambda}(x)$ of the operator $\mathcal{L}(20)$ are given by

$$
f_{\lambda}(x)=\frac{h_{\lambda}}{x-\lambda}
$$

where $h_{\lambda} \in \operatorname{Ker}(I-R V(\lambda))$ and it does not depend on $x$, besides, $\operatorname{dim} \operatorname{Ker}(I-$ $R V(\lambda))<\infty$; the eigenvalues $\lambda$ lie outside the $\operatorname{supp} a(x)$ and are zeroes of the function $D(\lambda)$ (36), $\overline{D(\lambda)}=D(\bar{\lambda})$; the order of the values $\lambda$ is $\operatorname{dim} \operatorname{Ker}(I-$ $R V(\lambda))$; the eigenvalues $\lambda \in \sigma_{p}(\mathcal{L})$ have the limiting points of the "absolutely continuous closure" [8] of the set $\sigma_{0}:=\{x \in \mathbb{R}: x \in \overline{\operatorname{supp} a(x)}\}$ as their accumulation points. The operator $R$ on the subspace $\operatorname{Ker}(I-R V(\lambda))$ is invertible.

P r o o f. Equality $\mathcal{L} f_{\lambda}(x)=\lambda f_{\lambda}(x)$ implies

$$
(x-\lambda) f_{\lambda}(x)-R \int_{\mathbb{R}} a(t) f_{\lambda}(t) d t=0,
$$

and for $h_{\lambda}(x)=(x-\lambda) f_{\lambda}(x)$ we obtain

$$
h_{\lambda}(x)-R \int_{\mathbb{R}} a(t) \frac{h_{\lambda}(t)}{t-\lambda} d t=0 .
$$

It is seen from the relation that $h_{\lambda}(x)=h_{\lambda}$ does not depend on $x$ and $[I-$ $R V(\lambda)] h_{\lambda}=0$. The finite dimensionality of $\operatorname{Ker}(I-R V(\lambda))$ provides the total continuity of the operator $R$. Taking into account that $(I-R V(\lambda))^{*}=I-V(\bar{\lambda}) R$ (in view of $\left.R=R^{*}, V^{*}(\lambda)=V(\bar{\lambda})\right)$ and using $\operatorname{det}(I-A B)=\operatorname{det}(I-B A)$ [17], we can prove that $\overline{D(\lambda)}=D(\bar{\lambda})$. There are no complex roots of $D(\lambda)(37)$ as $\mathcal{L}$ is selfadjoint.

It remains to prove that $R$ is not degenerate on $\operatorname{Ker}(I-R V(\lambda))$ in view of $\operatorname{dim} \operatorname{Ker}(I-R V(\lambda))<\infty$. If $h_{\lambda} \in \operatorname{Ker}(I-R V(\lambda))$, then $h_{\lambda}=R V(\lambda) h_{\lambda}$. Assuming the contrary, $R h_{\lambda}=0$, we obtain 


$$
\left\|h_{\lambda}\right\|^{2}=\left\langle h_{\lambda}, h_{\lambda}\right\rangle=\left\langle h_{\lambda}, R V(\lambda) h_{\lambda}\right\rangle=\left\langle R h_{\lambda}, V(\lambda) h_{\lambda}\right\rangle=0,
$$

and hence $h_{\lambda}=0$.

The singularities of $N(\lambda)(4)$ consist of an absolutely continuous part coinciding with $\sigma_{0}:=\{x \in \mathbb{R}: x \in \overline{\operatorname{supp} a(x)}\}(32),\left(\sigma_{0} \subseteq[a, b],-\infty<a<b<\infty\right.$, in view of the boundedness of $L_{0}$ ), and a discrete part which is the eigenvalues of $\mathcal{L}$ that are zeroes of $D(\lambda)(37)$ and lie outside $\{x \in \mathbb{R}: \overline{\operatorname{supp} a(x)}\}$. Thus,

$$
\sigma(\mathcal{L})=\sigma_{0} \cup \sigma_{p}
$$

where

$$
\sigma_{p} \stackrel{\text { def }}{=} \sigma_{p}(\mathcal{L})=\left\{\varkappa_{n} \in \mathbb{R}: D\left(\varkappa_{n}\right)=0\right\} .
$$

The sets $\sigma_{0}$ and $\sigma_{p}(44)$ do not intersect with each other except the limit points. Notice that if $\sigma_{0}$ consists of the finite (denumerable) number of not-intersecting segments from $[a, b]$, then the point spectrum (44) of the semiaxes $(-\infty, a)$ and $(b, \infty)$ is in the lacunas between these segments and probably has some limit points of these intervals as its accumulation points.

If $\varkappa_{n} \in \sigma_{p}(44)$, then the subspace

$$
F_{n} \stackrel{\text { def }}{=} \operatorname{Ker}\left(I-R V\left(\varkappa_{n}\right)\right)
$$

is nontrivial and finite-dimensional, $m_{n} \stackrel{\text { def }}{=} \operatorname{dim} F_{n}<\infty$.

Lemma 1. Suppose that

$$
\text { 1) } \overline{\operatorname{supp} a(x)}=[a, b] ; \quad 2) \bigcap_{x \in[a, b]} \operatorname{Ker} a(x)=\{0\} \text {. }
$$

Then the subspaces $F_{n}(45)$ do not intersect each other, $F_{n} \cap F_{k}=\{0\}(k \neq n)$.

$\mathrm{P}$ r o o f. Let there be a nonzero vector $h \in F_{k} \cap F_{n}(k \neq n)$. Then $R^{-1} h=V\left(\varkappa_{n}\right) h=V\left(\varkappa_{k}\right) h$, in view of the invertibility of $R$ on в $F_{n}$ (Theorem 14). Therefore, $\left\langle\left\{V\left(\varkappa_{n}\right)-V\left(\varkappa_{k}\right)\right\} h, h\right\rangle=0$, i. e.,

$$
\left(\varkappa_{n}-\varkappa_{k}\right) \int_{\mathbb{R}} \frac{\langle a(x) h, h\rangle}{\left(x-\varkappa_{n}\right)\left(x-\varkappa_{k}\right)} d x=0 .
$$

Since $\varkappa_{n} \neq \varkappa_{k}(n \neq k)$ and the integrand has a constant sign $\left(\varkappa_{n}, \varkappa_{k} \notin[a, b]\right)$, then $\langle a(x) h, h\rangle=0$ for all $x \in[a, b]$. Taking into account that $a(x) \geq 0$, we obtain $a(x) h=0$ for all $x \in[a, b]$, which contradicts condition 2) in (46). 
Corollary 2. If condition 1) (46) does not hold, then the statement of the lemma is not true. Let for $\operatorname{dim} E=1$ and $\overline{\operatorname{supp} a(x)}=[a, c] \cup[d, b](a<c<d<$ $b)$, the equation $1=R V(\lambda)$ always have two roots: $\varkappa_{1} \in(-\infty, a)$ and $\varkappa_{2} \in(c, d)$ as $R>0$, or $\varkappa_{1} \in(c, d)$ and $\varkappa_{2} \in(b, \infty)$ as $R<0$. Then the equality

$$
\int_{a}^{c} \frac{a(x) d x}{\left(x-\varkappa_{1}\right)\left(x-\varkappa_{2}\right)}+\int_{d}^{b} \frac{a(x) d x}{\left(x-\varkappa_{1}\right)\left(x-\varkappa_{2}\right)}=0
$$

does not imply $a(x)=0$.

Let $\varkappa_{n} \in \sigma_{p}(44)$. Then the eigenfunctions of the operator $\mathcal{L}$ (Theorem 14) have the form

$$
f_{\varkappa_{n}}^{s}(x)=\frac{h_{n}^{s}}{x-\varkappa_{n}} \quad\left(1 \leq s \leq m_{n}, n \in \mathbb{N}\right),
$$

where $\left\{h_{n}^{s}\right\}_{1}^{m_{n}}$ is the orthonormal basis in $F_{n}(45)$, and $D\left(\varkappa_{n}\right)=0(D(\lambda)$ is given by (36)). It is obvious that

$$
\left\langle f_{\varkappa_{n}}^{s}(x), f_{\varkappa_{n}}^{p}(x)\right\rangle_{L^{2}}=\left\langle V^{\prime}\left(\varkappa_{n}\right) h_{n}^{s}, h_{n}^{p}\right\rangle_{E} \quad\left(1 \leq s, p \leq m_{n}\right),
$$

where $V^{\prime}\left(\varkappa_{n}\right)$ is the value of the derivative of the function $V(\lambda)$ as $\lambda=\varkappa_{n}$. Show that $V^{\prime}\left(\varkappa_{n}\right)$ is non-degenerate on $F_{n}(45)$. Assuming the contrary, we suppose that there is a vector $h \in F_{n}$ such that $V^{\prime}\left(\varkappa_{n}\right) h=0$. Since $R^{-1} h=V\left(\varkappa_{n}\right) h$, then for all $f \in E$ we have

$$
\begin{gathered}
\left|\left\langle R^{-1} h, f\right\rangle\right|^{2}=\left|\left\langle V\left(\varkappa_{n}\right) h, f\right\rangle\right|^{2}=\left|\int_{\mathbb{R}} \frac{\langle a(x) h, f\rangle}{x-\varkappa_{n}} d x\right|^{2} \\
\leq \int_{\mathbb{R}} \frac{\langle a(x) h, h\rangle}{\left(x-\varkappa_{n}\right)^{2}} d x \cdot \int_{\mathbb{R}}\langle a(x) f, f\rangle d x=\left\langle V^{\prime}\left(\varkappa_{n}\right) h, h\right\rangle \int_{\mathbb{R}}\langle a(x) f, f\rangle d x=0 .
\end{gathered}
$$

The arbitrariness of $f \in E$ implies $R^{-1} h=0$. Consequently, $h=0$ ( $R$ is invertible on $F_{n}$ by Theorem 14 ).

Hence, the operator $P_{n} V^{\prime}\left(\varkappa_{n}\right) P_{n}$ is positive and invertible $\left(P_{n}\right.$ is an orthoprojector on $\left.F_{n}(45)\right)$. Therefore there exist

$$
M_{n} \stackrel{\text { def }}{=}\left\{P_{n} V^{\prime}\left(\varkappa_{n}\right) P_{n}\right\}^{-\frac{1}{2}} \quad(n \in \mathbb{N}),
$$

where $M_{n}>0$ and $\operatorname{rank} M_{n}=m_{n}$. Then the vector-functions

$$
f^{s}\left(\varkappa_{n}, x\right)=\frac{M_{n} h_{n}^{s}}{x-\varkappa_{n}} \quad\left(1 \leq s \leq m_{n}, n \in \mathbb{N}\right)
$$


form the orthonormal basis in $L_{\mathbb{R}}^{2}(E, a(x) d x)$ corresponding to the eigenvalue $\varkappa_{n}$ (for all $n \in \mathbb{N}$ ).

The functions $f^{s}\left(\varkappa_{n}, x\right)(48)$ are orthogonal for different $\varkappa_{n}$ as the eigenfunctions of the operator $\mathcal{L}$. Therefore,

$$
\int_{\mathbb{R}} \frac{1}{\left(x-\varkappa_{n}\right)\left(x-\varkappa_{l}\right)}\left\langle a(x) M_{n} h_{n}^{s}, M_{l} h_{l}^{k}\right\rangle d x=0,
$$

where $\left\{h_{n}^{s}\right\}_{1}^{m_{n}}$ and $\left\{h_{l}^{k}\right\}_{1}^{m_{l}}$ are orthonormal bases in $F_{n}$ and $F_{l}(44)$, respectively. Hence,

$$
M_{l}\left\{V\left(\varkappa_{n}\right)-V\left(\varkappa_{l}\right)\right\} M_{l}=0 \quad\left(\forall \varkappa_{n} \neq \varkappa_{l}\right) .
$$

Show that this equality holds automatically. Formula (45) implies $P_{n}=R V\left(\varkappa_{n}\right) P_{n}$ or $R^{-1} M_{n}=V\left(\varkappa_{n}\right) M_{n}$ in view of the invertibility of $R$ on $F_{n}$. Taking into account $M_{l} R^{-1}=M_{l} V\left(\varkappa_{l}\right)$, we have

$$
M_{l}\left\{V\left(\varkappa_{n}\right)-V\left(\varkappa_{l}\right)\right\} M_{n}=M_{l} R^{-1} M_{n}-M_{l} R^{-1} M_{n}=0 .
$$

Theorem 15. Suppose that the operator $R$ is of trace-class; the representing measure $d F(x)$ of the function $V(\lambda)(2)$ is absolutely continuous $(21) ;\left\{\varkappa_{n}\right\}_{1}^{\infty}$ are the set of zeroes of the function $D(\lambda)(37), D\left(\varkappa_{n}\right)=0$, and $\varkappa_{n} \notin \sigma_{0}$.

Then the function $N(\lambda)$ (4) is given by

$$
N(\lambda)=R+\sum_{n=1}^{\infty} \frac{M_{n}^{2}}{\varkappa_{n}-\lambda}+\int_{\sigma_{0}} \frac{b(x)}{x-\lambda} d x,
$$

where $b(x) \geq 0, \overline{\operatorname{supp} b(x)}=\sigma_{0}$, and $M_{n}$ are given by (47). Moreover,

$$
\int_{\sigma_{0}} b(x) d x<\infty ; \quad \sum_{n=1}^{\infty} M_{n}^{2}<\infty .
$$

P r o o f. Let us turn back to representation 4) (5) of the function $N(\lambda)$ (4). Then the absolute continuity of $d \sigma(t)$ on $\sigma_{0}$ follows from (32) and the KatoRosenblum theorem [2]. It is necessary to prove that discontinuities of the measure $d \sigma(t)$ at the points $\varkappa_{n}$ coinside with $M_{n}^{2}$.

Write the Laurent expansion of $N(\lambda)(4)$ at the point $\varkappa_{n} \in \sigma_{p}(44)$,

$$
N(\lambda)=\frac{\sigma_{n}}{\varkappa_{n}-\lambda}+R+\ldots,
$$


where $\sigma_{n} \geq 0$, and substitute the obtained equality into the identity $N(\lambda)=$ $R+R V(\lambda) N(\lambda)$. Then

$$
\frac{\sigma_{n}}{\varkappa_{n}-\lambda}+R+\ldots=R+R V(\lambda)\left(\frac{\sigma_{n}}{\varkappa_{n}-\lambda}+R+\ldots\right) .
$$

Multiplying the last equality by $\left(\varkappa_{n}-\lambda\right)$ and passing to limit as $\lambda \rightarrow \varkappa_{n}$, we obtain that $\sigma_{n}=R V\left(\varkappa_{n}\right) \sigma_{n}\left(V\left(\varkappa_{n}\right)\right.$ exist since $\left.\varkappa_{n} \notin \sigma_{0}\right)$. Show that $\sigma_{n}$ : $F_{n} \rightarrow F_{n}$ and $\sigma_{n} F_{n}^{\perp}=0$. The definition of $F_{n}(45)$ implies $P_{n}=R V\left(\varkappa_{n}\right) P_{n}\left(P_{n}\right.$ is an orthoprojector on $F_{n}$ ). Then

$$
\sigma_{n}=R V\left(\varkappa_{n}\right) \sigma_{n}=R V\left(\varkappa_{n}\right)\left[P_{n}+P_{n}^{\perp}\right] \sigma_{n}=P_{n} \sigma_{n}+R V\left(\varkappa_{n}\right) P_{n}^{\perp} \sigma_{n},
$$

where $P_{n}^{\perp}=I-P_{n}$. Therefore, $P_{n}^{\perp} \sigma_{n}=R V\left(\varkappa_{n}\right) P_{n}^{\perp} \sigma_{n}$, or $\left(I-R V\left(\varkappa_{n}\right)\right) P_{n}^{\perp} \sigma_{n}$ $=0$. Thus, $P_{n}^{\perp} \sigma_{n} f \in F_{n}$ for all $f \in F_{n}$ and $P_{n}^{\perp} \sigma_{n} P_{n}=0$, which proves that $\sigma_{n}$ : $F_{n} \rightarrow F_{n}$ and $\left.\sigma_{n}\right|_{F_{n}^{\perp}}=0$, in view of the self-adjointness of $\sigma_{n}$.

Now we have to prove that $\sigma_{n}=M_{n}^{2}(47)$. First, we multiply the equality $R=(I-R V(\lambda)) N(\lambda)$ from the right by $P_{n}$. Then, taking into account the invertibility of $R$ on $F_{n}$, we obtain

$$
\frac{R^{-1}-V(\lambda)}{\varkappa_{n}-\lambda}\left(\varkappa_{n}-\lambda\right) N(\lambda) P_{n}=P_{n}
$$

Using the Laurent expansion of $N(\lambda)$ at the point $\lambda=\varkappa_{n}$ and passing to limit as $\lambda \rightarrow \varkappa_{n}$, we have $V^{\prime}\left(\varkappa_{n}\right) \sigma_{n} P_{n}=P_{n}$, which gives us $M_{n}^{-2} \sigma_{n}=P_{n}\left(\sigma_{n} P_{n}=P_{n} \sigma_{n}\right)$. The convergence of the integral and the series in (50) follows from the finiteness of variation of the measure $d \sigma(t)$ at representation 4) (5) of the function $N(\lambda)$.

VII. Find the resolution of the identity $E_{\lambda}^{a}$ corresponding to the absolutely continuous part of $\mathcal{L}^{a}$ of the operator $\mathcal{L}$. Then

$$
\frac{d}{d \lambda}\left\langle E_{\lambda}^{a} f, f\right\rangle=\lim _{\varepsilon \rightarrow+0}\langle\delta(\lambda, \varepsilon) f, f\rangle
$$

where $\delta(\lambda, \varepsilon)(7)$ corresponds to the operator $\mathcal{L}$. Using (41), we obtain

$$
\begin{aligned}
&\langle\delta(\lambda, \varepsilon) f, f\rangle= \frac{1}{2 \pi i}\left\{\left\langle R_{\mathcal{L}}(\lambda+i \varepsilon) f, f\right\rangle-\left\langle R_{\mathcal{L}}(\lambda-i \varepsilon) f, f\right\rangle\right\}=\frac{1}{2 \pi i}\left\langle\frac{f(x)}{x-\lambda-i \varepsilon}\right. \\
&\left.+\frac{N(\lambda+i \varepsilon)}{x-\lambda-i \varepsilon} \int_{\mathbb{R}} \frac{a(t)}{t-\lambda-i \varepsilon} f(t) d t, f(x)\right\rangle-\frac{1}{2 \pi i}\left\langle\frac{f(x)}{x-\lambda+i \varepsilon}-\frac{N(\lambda-i \varepsilon)}{x-\lambda+i \varepsilon}\right. \\
&\left.\quad \times \int_{\mathbb{R}} \frac{a(t)}{t-\lambda+i \varepsilon} f(t) d t, f(x)\right\rangle=A_{\varepsilon}+B_{\varepsilon}+\overline{B_{\varepsilon}},
\end{aligned}
$$


where,

$$
\begin{gathered}
A_{\varepsilon} \stackrel{\text { def }}{=} \frac{1}{\pi} \int_{\mathbb{R}} \frac{\varepsilon}{(x-\lambda)^{2}+\varepsilon^{2}}\langle a(x) f(x), f(x)\rangle d x, \\
B_{\varepsilon} \stackrel{\text { def }}{=} \frac{1}{\pi} \int_{\mathbb{R}}\left\langle N(\lambda+i \varepsilon) \int_{\mathbb{R}} \frac{a(t)}{t-\lambda-i \varepsilon} f(t) d t, \int_{\mathbb{R}} \frac{a(x)}{x-\lambda+i \varepsilon} f(x) d x\right\rangle .
\end{gathered}
$$

Lemma 2. For all $\lambda \in \mathbb{R}$, the formula

$$
\begin{aligned}
A_{0}+B_{0}+\overline{B_{0}} & =\langle a(\lambda) f(\lambda), f(\lambda)\rangle-\pi^{2}\left\langle a(\lambda) N_{-}(\lambda) a(\lambda) f(\lambda), N_{-}(\lambda) a(\lambda) f(\lambda)\right\rangle \\
& +\operatorname{Re}\left\langle\left[N_{1}(\lambda)+N_{-}(\lambda)\right] a(\lambda) f(\lambda), \int_{\mathbb{R}} \frac{a(x)}{t-\lambda} f(t) d t\right\rangle \\
+ & \left\langle a(\lambda) N_{-}(\lambda) \int_{\mathbb{R}} \frac{a(t)}{t-\lambda} f(t) d t, N_{-}(\lambda) \int_{\mathbb{R}} \frac{a(t)}{t-\lambda} f(t) d t\right\rangle
\end{aligned}
$$

is true, where $A_{0}\left(B_{0}\right)=\left.A_{\varepsilon}\left(B_{\varepsilon}\right)\right|_{\varepsilon=+0}$, and $N_{ \pm}(\lambda)$ are the boundary values on $\mathbb{R}$ from $\mathbb{C}_{ \pm}$of the function $N(\lambda)(4)$.

P r o o f. The property of the Poisson kernel $[14,15]$ yields

$$
A_{\varepsilon} \rightarrow A_{0}=\langle a(\lambda) f(\lambda), f(\lambda)\rangle \quad(\varepsilon \rightarrow+0) .
$$

Using the Sokhotsky formulas [16], we have

$$
\begin{gathered}
B_{0}=-2 \pi i\left\langle N_{+}(\lambda)\left\{\frac{1}{2} a(\lambda) f(\lambda)+\frac{1}{2 \pi i} \int_{\mathbb{R}} \frac{a(t)}{t-\lambda} f(t) d t\right\},\right. \\
\left.\left\{-\frac{1}{2} a(\lambda) f(\lambda)+\frac{1}{2 \pi i} \int_{\mathbb{R}} \frac{a(t)}{t-\lambda} f(t) d t\right\}\right\rangle .
\end{gathered}
$$

Finally, after elementary transformations, taking into account (32), we obtain (51).

Theorem 16. Suppose that the suppositions of Theorem 6 take place. Then for the identity resolution $E_{\lambda}^{a}$ of the absolutely continuous part $\mathcal{L}^{a}$ of the operator $\mathcal{L}(20)$, the representation

$$
\begin{aligned}
\frac{d}{d \lambda}\left\langle E_{\lambda}^{a} f, f\right\rangle & =\left\langle a(\lambda)\left\{\frac{1}{2}\left(I+S^{+}(\lambda)\right) f(\lambda)+N_{+}(\lambda) \int_{\mathbb{R}} \frac{a(t)}{t-\lambda} f(t) d t\right\},\right. \\
& \left.\left\{\frac{1}{2}\left(I+S^{+}(\lambda)\right) f(\lambda)+N_{+}(\lambda) \int_{\mathbb{R}} \frac{a(t)}{t-\lambda} f(t) d t\right\}\right\rangle
\end{aligned}
$$


is true, where $S^{+}(\lambda)$ is the scattering matrix (25), and $N_{+}(\lambda)$ is the boundary value on $\mathbb{R}$ from $\mathbb{C}_{+}$of the function $N(\lambda)$ (4).

P r o o f. Since $N_{-}(\lambda)=N_{+}(\lambda)-2 \pi i N_{+}(\lambda) a(\lambda) N_{-}(\lambda)(32)$, then the third summand from (51) is given by

$$
\begin{gathered}
\frac{1}{2}\left\langle\left[N_{+}(\lambda)+N_{-}(\lambda)\right] \int_{\mathbb{R}} \frac{a(t)}{t-\lambda} f(t) d t, a(\lambda) f(\lambda)\right\rangle=\left\langle N_{+}(\lambda) \int_{\mathbb{R}} \frac{a(t)}{t-\lambda} f(t) d t,\right. \\
a(\lambda) f(\lambda)\rangle+\left\langle a(\lambda) N_{-}(\lambda) \int_{\mathbb{R}} \frac{a(\lambda)}{t-\lambda} f(t) d t, \pi i N_{-}(\lambda) a(\lambda) f(\lambda)\right\rangle .
\end{gathered}
$$

Substituting this expression in (51) and taking into account that $S(\lambda) N_{+}(\lambda)=$ $N_{-}(\lambda)(35)$ and $\left.S^{*}(\lambda) a(\lambda) S(\lambda)=a(\lambda) 1\right)(33)$, we obtain

$$
\begin{aligned}
& A_{0}+B_{0}+\overline{B_{0}}=\langle a(\lambda) f(\lambda), f(\lambda)\rangle-\left\langle a(\lambda) \pi i N_{+}(\lambda) a(\lambda) f(\lambda), \pi i N_{+}(\lambda) a(\lambda) f(\lambda)\right\rangle \\
&+\left\langle N_{+}(\lambda) \int_{\mathbb{R}} \frac{a(t)}{t-\lambda} f(t) d t, a(\lambda) f(\lambda)\right\rangle+\left\langle a(\lambda) N_{+}(\lambda) \int_{\mathbb{R}} \frac{a(t)}{t-\lambda} f(t) d t\right. \\
&\left.\pi i N_{+}(\lambda) a(\lambda) f(\lambda)\right\rangle+\left\langle a(\lambda) f(\lambda), N_{+}(\lambda) \int_{\mathbb{R}} \frac{a(t)}{t-\lambda} f(t) d t\right\rangle \\
&+\left\langle a(\lambda) \pi i N_{+}(\lambda) a(\lambda) f(\lambda), N_{+}(\lambda) \int_{\mathbb{R}} \frac{a(t)}{t-\lambda} f(t) d t\right\rangle \\
&+\left\langle a(\lambda) N_{+}(\lambda) \int_{\mathbb{R}} \frac{a(t)}{t-\lambda} f(t) d t, N_{+}(\lambda) \int_{\mathbb{R}} \frac{a(t)}{t-\lambda} f(t) d t\right\rangle .
\end{aligned}
$$

Hence, after elementary transformations we obtain

$$
\begin{gathered}
A_{0}+B_{0}+\overline{B_{0}}=\left\langle a(\lambda)\left\{f(\lambda)+\pi i N_{+}(\lambda) a(\lambda) f(\lambda)+N_{+}(\lambda) \int_{\mathbb{R}} \frac{a(t)}{t-\lambda} f(t) d t\right\},\right. \\
\left.\left\{f(\lambda)+\pi i N_{+}(\lambda) a(\lambda) f(\lambda)+N_{+}(\lambda) \int_{\mathbb{R}} \frac{a(t)}{t-\lambda} f(t) d t\right\}\right\rangle
\end{gathered}
$$

which gives us (52) in view of (25). 


\section{Equation of the Inverse Scattering Problem}

VIII. Associate every function $f(x) \in L_{\mathbb{R}}^{2}(E, d F(x))(18)$ with the Fourier coefficients,

$$
f^{s}\left(\varkappa_{n}\right) \stackrel{\text { def }}{=}\left\langle f(x), f^{s}\left(\varkappa_{n}, x\right)\right\rangle \quad\left(1 \leq s \leq m_{n}, n \in \mathbb{N}\right)
$$

corresponding to the discrete spectrum $\sigma_{p}(\mathcal{L})(44)$ of the operator $\mathcal{L}$, where $f^{s}\left(\varkappa_{n}, x\right)$ are given by $(48)$. Then

$$
f^{s}\left(\varkappa_{n}\right)=\left\langle\int_{\mathbb{R}} \frac{a(x)}{x-\varkappa_{n}} f(x) d x, M_{n} h_{n}^{s}\right\rangle,
$$

where $\left\{h_{n}^{s}\right\}_{1}^{m_{n}}$ are the orthonormal basis in $F_{n}(45)$, and $M_{n}$ are given by (47).

In the case of absolutely continuous spectrum of the operator $\mathcal{L}$,

$$
\tilde{f}(\lambda) \stackrel{\text { def }}{=} \frac{1}{2}\left(I+S^{+}(\lambda)\right) f(\lambda)+N_{+}(\lambda) \int_{\mathbb{R}} \frac{a(t)}{t-\lambda} f(t) d t
$$

are the "Fourier coefficients" of the function $f(x) \in L_{\mathbb{R}}^{2}(E, d F(x))$ by Theorem 16. The space $L_{\mathbb{R}}^{2}(E, d F(x))$ is the orthogonal sum of two subspaces, the first of which is the eigenfunctions (associated states) of the operator $\mathcal{L}$, and the second subspace corresponds to the absolutely continuous spectrum of $\mathcal{L}$. Write the Parseval equality [4],

$$
\langle f(x), g(x)\rangle_{L_{\mathbb{R}}^{2}(E, d F(x))}=\sum_{n=1}^{\infty} \sum_{s=1}^{m_{n}} f^{s}\left(\varkappa_{n}\right) \overline{g^{s}\left(\varkappa_{n}\right)}+\langle\tilde{f}(\lambda), \tilde{g}(\lambda)\rangle_{L_{\mathbb{R}}^{2}(E, d F(\lambda))},
$$

where $f, g \in L_{\mathbb{R}}^{2}(E, d F(x))$. (54) implies

$$
\sum_{s=1}^{m_{n}} f^{s}\left(\varkappa_{n}\right) \overline{g^{s}\left(\varkappa_{n}\right)}=\int_{\mathbb{R}} d x \int_{\mathbb{R}} d y\left\langle M_{n}^{2} \frac{a(x)}{x-\varkappa_{n}} f(x), \frac{a(y)}{y-\varkappa_{n}} g(y)\right\rangle
$$

since $\left\{h_{n}^{s}\right\rangle_{1}^{m_{n}}$ is the orthonormal basis in $F_{n}$. Using

$$
\frac{1}{\left(x-\varkappa_{n}\right)\left(y-\varkappa_{n}\right)}=\frac{1}{x-y}\left[\frac{1}{y-\varkappa_{n}}-\frac{1}{x-\varkappa_{n}}\right] \text {, }
$$

we obtain

$$
\sum_{n=1}^{\infty} \sum_{s=1}^{m_{n}} f^{s}\left(\varkappa_{n}\right) \overline{g^{s}\left(\varkappa_{n}\right)}=-\int_{\mathbb{R}} d x \int_{\mathbb{R}} d y\left\langle\frac{P(x)-P(y)}{x-y} a(x) f(x), a(y) f(y)\right\rangle,
$$


where

$$
P(x) \stackrel{\text { def }}{=} \sum_{n=1}^{\infty} \frac{M_{n}^{2}}{x-\varkappa_{n}} .
$$

By the condition of (50), the series of (57) converges.

Equality (55) implies

$$
\begin{aligned}
B \stackrel{\text { def }}{=}\langle\tilde{f}(\lambda), \tilde{g}(\lambda)\rangle_{L_{\mathbb{R}}^{2}(E, d F(\lambda))}=\int_{\mathbb{R}}\left\langlea ( \lambda ) \left\{\frac{1}{2}\left(I+S^{+}(\lambda)\right) f(\lambda)\right.\right. \\
\left.\left.+N_{+}(\lambda) \int_{\mathbb{R}} \frac{a(t)}{t-\lambda} f(t) d t\right\},\left\{\frac{1}{2}\left(I+S^{+}(\lambda)\right) g(\lambda)+N_{+}(\lambda) \int_{\mathbb{R}} \frac{a(t)}{t-\lambda} g(t) d t\right\}\right\rangle \\
=\frac{1}{4} \int_{\mathbb{R}}\left\langle\left[2 a(\lambda)+a(\lambda) S^{+}(\lambda)+\left(S^{+}(\lambda)\right)^{*} a(\lambda)\right] f(\lambda), g(\lambda)\right\rangle d \lambda \\
+\frac{1}{2} \int_{\mathbb{R}}\left\langle a(\lambda)\left(I+S^{+}(\lambda)\right) f(\lambda), N_{+}(\lambda) \int_{\mathbb{R}} \frac{a(t)}{t-\lambda} g(t) d t\right\rangle \\
+\frac{1}{2} \int_{\mathbb{R}}\left\langle a(\lambda) N_{+}(\lambda) \int_{\mathbb{R}} \frac{a(t)}{t-\lambda} f(t) d t,\left(I+S^{+}(\lambda)\right) g(\lambda)\right\rangle d \lambda \\
+\int_{\mathbb{R}}\left\langle N_{-}(\lambda) a(\lambda) N_{+}(\lambda) \int_{\mathbb{R}} \frac{a(t)}{t-\lambda} f(t) d t, \int_{\mathbb{R}} \frac{a(t) g(t)}{t-\lambda} d t\right\rangle d \lambda .
\end{aligned}
$$

Using (26), (32), (33), we obtain

$$
\begin{aligned}
B=\langle f(x), g(x)\rangle_{L_{\mathbb{R}}^{2}(E, d F(x))}+\frac{1}{4} \int_{\mathbb{R}}\left\langle a(\lambda)\left[S^{+}(\lambda)+S(\lambda)-2 I\right] f(\lambda), g(\lambda)\right\rangle d \lambda \\
+\frac{1}{2} \int_{\mathbb{R}}\left\langle N_{-}(\lambda)\left[I+S^{*}(\lambda)\right] a(\lambda) f(\lambda), \int_{\mathbb{R}} \frac{a(t)}{t-\lambda} g(t) d t\right\rangle d \lambda \\
+\frac{1}{2} \int_{\mathbb{R}}\left\langle a(\lambda)[I+S(\lambda)] N_{+}(\lambda) \int_{\mathbb{R}} \frac{a(t)}{t-\lambda} f(t) d t, g(\lambda)\right\rangle d \lambda \\
+\frac{1}{2 \pi i} \int_{\mathbb{R}}\left\langle\left[N_{+}(\lambda)-N_{-}(\lambda)\right] \int_{\mathbb{R}} \frac{a(t)}{t-\lambda} f(t) d t, \int_{\mathbb{R}} \frac{a(t)}{t-\lambda} g(t) d t\right\rangle d \lambda .
\end{aligned}
$$


Taking into account (22), (25), and (35), we have

$$
\begin{gathered}
B=\langle f(x), g(x)\rangle_{L_{\mathbb{R}}^{2}(E, d F(x))}+\frac{\pi i}{2} \int_{\mathbb{R}}\left\langle a(\lambda)[I-S(\lambda)] N_{+}(\lambda) a(\lambda) f(\lambda), g(\lambda)\right\rangle d \lambda \\
-\frac{1}{2} \int_{\mathbb{R}}\left\langle a(\lambda) \int_{\mathbb{R}} \frac{(I+S(t)) N_{+}(t)-(I+S(\lambda)) N(\lambda)}{t-\lambda} a(t) f(t) d t, g(\lambda)\right\rangle d \lambda \\
\quad+\frac{1}{2 \pi i} \int_{\mathbb{R}}\left\langle[I-S(\lambda)] N_{+}(\lambda) \int_{\mathbb{R}} \frac{a(t)}{t-\lambda} f(t) d t, \int_{\mathbb{R}} \frac{a(t)}{t-\lambda} g(t) d t\right\rangle d \lambda .
\end{gathered}
$$

The last integral from this sum may be written in the form

$$
C \stackrel{\text { def }}{=} \frac{1}{2 \pi i} \int_{\mathbb{R}} d x \int_{\mathbb{R}} \frac{d \lambda}{x-\lambda}\left\langle(I-S(\lambda)) N_{+}(\lambda) \int_{\mathbb{R}} \frac{a(t)}{t-\lambda} f(t) d t, a(x) g(x)\right\rangle .
$$

Using the Poincare-Bertrand formula [16] for rearranging the order of integration in improper integrals,

$$
\frac{1}{\pi i} \int_{\mathbb{R}} \frac{d \tau}{\tau-t} \int_{\mathbb{R}} \frac{\varphi(\tau, \xi)}{\xi-\tau} d \xi=\pi i \varphi(t, t)+\int_{\mathbb{R}} d \xi \int_{\mathbb{R}} \frac{\varphi(\tau, \xi)}{(\tau-t)(\xi-\tau)} d \tau,
$$

we obtain

$$
\begin{gathered}
\frac{1}{\pi i} \int_{\mathbb{R}} \frac{d \lambda}{\lambda-x} \int_{\mathbb{R}} \frac{(I-S(\lambda)) N_{+}(\lambda)}{t-\lambda} a(t) f(t) d t=\pi i[I-S(x)] N_{+}(x) a(x) f(x) \\
+\frac{1}{\pi i} \int_{\mathbb{R}} d t \int_{\mathbb{R}} \frac{(I-S(\lambda)) N_{+}(\lambda)}{(t-\lambda)(\lambda-x)} a(t) f(t) d \lambda .
\end{gathered}
$$

Therefore,

$$
\begin{gathered}
C=-\frac{\pi i}{2} \int_{\mathbb{R}}\left\langle[I-S(x)] N_{+}(x) a(x) f(x), a(x) g(x)\right\rangle d x \\
+\frac{1}{2 \pi i} \int_{\mathbb{R}} d x\left\langle\int_{\mathbb{R}} \frac{M(x)-M(t)}{x-t} a(t) f(t) d t, a(x) g(x)\right\rangle,
\end{gathered}
$$

where

$$
M(x) \stackrel{\text { def }}{=} \int_{\mathbb{R}} \frac{(I-S(\lambda)) N_{+}(\lambda)}{\lambda-x} d \lambda
$$


Finally, we have

$$
\begin{gathered}
\langle\tilde{f}(\lambda), \tilde{g}(\lambda)\rangle_{L_{\mathbb{R}}^{2}(E, d F(\lambda))}=\langle f(x), g(x)\rangle_{L_{\mathbb{R}}^{2}(E, d F(x))} \\
-\frac{1}{2} \int_{\mathbb{R}}\left\langle a(\lambda) \int_{\mathbb{R}} \frac{[I+S(t)] N_{+}(t)-[I+S(\lambda)] N_{+}(\lambda)}{t-\lambda} a(t) f(t) d t, g(\lambda)\right\rangle d \lambda \\
+\frac{1}{2 \pi i} \int_{\mathbb{R}}\left\langle a(\lambda) \int_{\mathbb{R}} \frac{M(t)-M(\lambda)}{t-\lambda} a(t) f(t) d t, g(\lambda)\right\rangle d \lambda .
\end{gathered}
$$

Substituting the obtained expressions in the Parseval equality (56), we have

$$
\begin{gathered}
-\int_{\mathbb{R}} d \lambda\left\langle a(\lambda) \int_{\mathbb{R}} \frac{P(x)-P(\lambda)}{x-\lambda} a(x) f(x) d x, g(\lambda)\right\rangle \\
-\frac{1}{2} \int_{\mathbb{R}} d \lambda\left\langle a(\lambda) \int_{\mathbb{R}} \frac{[I+S(x)] N_{+}(x)-[I+S(\lambda)] N_{+}(\lambda)}{x-\lambda} a(x) f(x) d x, g(\lambda)\right\rangle \\
+\frac{1}{2 \pi i} \int_{\mathbb{R}} d \lambda\left\langle a(\lambda) \int_{\mathbb{R}} \frac{M(x)-M(\lambda)}{x-\lambda} a(x) f(x) d x, g(\lambda)\right\rangle=0 .
\end{gathered}
$$

Let

$$
G(\lambda) \stackrel{\text { def }}{=}-P(\lambda)-\frac{1}{2}[I+S(\lambda)] N_{+}(\lambda)+\frac{1}{2 \pi i} \int_{\mathbb{R}} \frac{[I-S(x)] N_{+}(x)}{x-\lambda} d x .
$$

Then the last equality takes the form

$$
\int_{\mathbb{R}}\left\langle a(\lambda) \int_{\mathbb{R}} \frac{G(x)-G(\lambda)}{x-\lambda} a(x) f(x) d x, g(\lambda)\right\rangle d \lambda=0,
$$

that, in view of the arbitrariness of $g(x) \in L_{\mathbb{R}}^{2}(E, a(x) d x)$, gives

$$
\int_{\mathbb{R}} \frac{G(x)-G(\lambda)}{x-\lambda} a(x) f(x) d x=0
$$

for all $f(x) \in L_{\mathbb{R}}^{2}(E, a(x) d x)$. This implies

$$
\int_{\mathbb{R}}\left\langle a(\lambda) f(\lambda), \frac{G(x)-G(\lambda)}{x-\lambda} h\right\rangle=0
$$


for an arbitrary $h \in E$, in view of the self-adjointness of $G(\lambda)(59)$, since $S(\lambda) N_{+}(\lambda)=$ $N_{-}(\lambda)(35)$. Thus,

$$
\frac{G(x)-G(\lambda)}{x-\lambda} h=0 \quad(\forall x, \lambda)
$$

and $G(\lambda)$ is a constant operator

$$
G(\lambda)=B\left(=B^{*}\right)
$$

Since

$$
G(\lambda)=-P(\lambda)-N_{+}(\lambda)+\frac{1}{2}[I-S(\lambda)] N_{+}(\lambda)+\frac{1}{2 \pi i} \int_{\mathbb{R}} \frac{[I-S(x)] N_{+}(x)}{x-\lambda} d x,
$$

then

$$
G(\lambda)=-P(\lambda)-N_{+}(\lambda)+\frac{1}{2 \pi i} \int_{\mathbb{R}} \frac{[I-S(x)] N_{+}(x)}{x-\lambda-i 0} d x \quad(\lambda \in \mathbb{R})
$$

in view of the Sokhotsky formula [16], therefore

$$
G(\lambda)=-P(\lambda)-N(\lambda)+\frac{1}{2 \pi i} \int_{\mathbb{R}} \frac{[I-S(x)] N_{+}(x)}{x-\lambda} d x \quad\left(\lambda \in \mathbb{C}_{+}\right) .
$$

Let $\lambda=i y(y>0)$, then the first and the third members of this sum converge to zero as $y \rightarrow+\infty$ and $N(i y) \rightarrow R(y \rightarrow \infty)$ in view of 4$)(5)$, and we get $B=-R$.

Theorem 17. Let $f, g \in L_{\mathbb{R}}^{2}(E, d F(x))$, where $F(x)$ is absolutely continuous (21), $R$ is of trace-class, $\varphi$ is a Hilbert-Schmidt operator, and $f^{s}\left(\varkappa_{n}\right), \tilde{f}(\lambda)$, and $g^{s}\left(\varkappa_{n}\right), \tilde{g}(\lambda)$, are the corresponding Fourier coefficients (54), (55) of these functions.

For the Parseval equality (56) to be fulfilled, it is necessary and sufficient that the relation

$$
R-P(\lambda)-\frac{1}{2}[I+S(\lambda)] N_{+}(\lambda)+\frac{1}{2 \pi i} \int_{\mathbb{R}} \frac{[I-S(x)] N_{+}(x)}{x-\lambda} d x=0 \quad(\lambda \in \mathbb{R})
$$

hold, where $P(\lambda)$ is given by $(57) ; \varkappa_{n}$ are zeroes of $D(\lambda)(37) ; M_{n}$ are the finitedimensional positive invertible operators (47) such that series (50) converges; $N_{+}(\lambda)$ are the boundary values on $\mathbb{R}$ from $\mathbb{C}_{+}$of the function $N(\lambda)(4) ; S(\lambda)$ is the scattering matrix (22).

Corollary 3. Taking into account $S(\lambda) N_{+}(\lambda)=N_{-}(\lambda)(35)$, we write (61) in the form

$$
R-F(\lambda)-\frac{1}{2}\left[N_{+}(\lambda)+N_{-}(\lambda)\right]+\frac{1}{2 \pi i} \int_{\mathbb{R}} \frac{N_{+}(x)-N_{-}(x)}{x-\lambda} d x=0 .
$$


In view of the Sokhotsky formula [16], (49) implies

$$
N_{ \pm}(\lambda)=R+\sum_{n=1}^{\infty} \frac{M_{n}^{2}}{\varkappa_{n}-\lambda} \pm \pi i b(\lambda)+\int_{\mathbb{R}} \frac{b(x)}{x-\lambda} d x \quad(\lambda \in \mathbb{R}) .
$$

Substituting $N_{ \pm}(\lambda)$ (63) into (62), we obtain an identity.

Relation (61) should be considered as an equation with respect to $N_{+}(\lambda)$ which is an analogue of the Marchenko equation for the inverse scattering problem [4-7]. Besides, we ought to search solution (61) in the class of Nevanlinna functions 4) (5).

Let $N_{+}(\lambda)$ be the solution of equation $(61)$, and $N(\lambda)$ be its analytic continuation into $\mathbb{C}_{+}$in view of (60). Continue $N(\lambda)$ into $\mathbb{C}_{-}$in a natural way, then, using the Sokhotsky formula and (61), we obtain

$$
N_{ \pm}(\lambda)=R-F(\lambda) \pm \frac{1}{2}(I-S(\lambda)) N_{+}(\lambda)+\frac{1}{2 \pi i} \int_{\mathbb{R}} \frac{[I-S(x)] N_{+}(x)}{x-\lambda} d x .
$$

This implies the statement.

Theorem 18. Suppose that the suppositions of Theorem 6 are true, $N_{+}(\lambda)$ is the solution of equation $(61), N(\lambda)$ is its holomorphic extension into $\mathbb{C}_{+}$, and $N_{-}(\lambda)$ is a boundary value on $\mathbb{R}$ from $\mathbb{C}_{-}$after the natural extension of $N(\lambda)$ into $\mathbb{C}_{-}$. Then the Riemann boundary value problem $S(\lambda) N_{+}(\lambda)=N_{-}(\lambda)$ (38) takes place. If

$$
\frac{1}{2 i}(I-S(\lambda)) N_{+}(\lambda) \geq 0 \quad(\lambda \in \mathbb{R}),
$$

then $N_{-}(\lambda)=N_{+}^{*}(\lambda)$.

P r o o f. The Riemann problem follows from (64) after the subtraction of $N_{-}(\lambda)-N_{+}(\lambda)$. Condition (65) is natural in view of (32). The fact that (65) implies $N_{-}(\lambda)=N_{+}^{*}(\lambda)$ is obvious in view of $(64)$.

\section{Inverse Problem}

IX. Let $\operatorname{dim} E=1$ and $\sigma_{0}$ be the segment $\sigma_{0}=[a, b](-\infty<a<b<\infty)$. The discrete spectrum $\sigma_{p}(44)$ is formed by the zeroes of the equation $1=R V(\lambda)$. Taking into account monotonicity of $V(\lambda)$ as $\lambda \in \mathbb{R} \backslash[a, b]$, we see that the root $1=R V(\varkappa)$ is unique since $\varkappa \in(-\infty, a)$ for $R>0$, and $\varkappa \in(b, \infty)$ for $R<0$. Thus, $\sigma_{p}=\{\varkappa \notin[a, b]\}$.

Corollary 4. The set $\sigma_{p}$ can be empty depending on the number $R$ and on the behavior of the representing measure $d F(x)=a(x) d x$ of the function $V(\lambda)(2)$ at 
the end points of the segment $[a, b]$. If, for example, $a(x)=\alpha(x-a)(\alpha>0)$, then

$$
V(\lambda)=\alpha(b-a)+\alpha(\lambda-a) \int_{a}^{b} \frac{d x}{x-\lambda} .
$$

The equation $1=R V(\lambda)$ has no roots when $R>\{\alpha(b-a)\}^{-1}$ as $V(a-0)=$ $\alpha(b-a)$.

In this case, the Riemann boundary value problem (34) can be written as

$$
S(\lambda) \frac{\lambda+i}{\lambda-i} \frac{\lambda-\varkappa}{\lambda+i}\left(1-R V_{+}(\lambda)\right)^{-1}=\frac{\lambda-\varkappa}{\lambda-i}\left(1-R V_{-}(\lambda)\right)^{-1} .
$$

Each function $\frac{\lambda-\varkappa}{\lambda \pm i}\left(1-R V_{ \pm}(\lambda)\right)^{-1}$ is holomorphically extendable in $\mathbb{C}_{ \pm}$and has no zeroes and singularities in $\overline{\mathbb{C}_{ \pm}}$, besides, it tends to 1 at the infinity. Using the standard method [16] of the solution of the boundary problem (66) and the Sokhotsky formulas [16], we obtain

$$
\frac{\lambda-\varkappa}{\lambda+i}(1-R V(\lambda))=\exp \left\{-\frac{1}{2 \pi i} \int_{\mathbb{R}}^{\ln \left[S(x) \frac{x+i}{x-i}\right]} \frac{x-\lambda}{x} d x\right\} \quad\left(\lambda \in \mathbb{C}_{+}\right) .
$$

Let

$$
\psi(x)=\frac{1}{\pi} \operatorname{arctg} \frac{1}{x} \quad\left(x \in \mathbb{R},-\frac{1}{2} \leq \psi(x) \leq \frac{1}{2}\right) .
$$

Then, taking into account (39), we have

$$
S(x) \frac{x+i}{x-i}=\exp \{-2 \pi i(\xi(x)-\psi(x))\},
$$

where $\xi(x)$ is the function of spectral shift (39). Equality (67) can be written in the form

$$
(1-R V(\lambda))^{-1}=\frac{\lambda+i}{\lambda-\varkappa} \exp \left\{\int_{\mathbb{R}} \frac{\xi(x)-\psi(x)}{x-\lambda} d x\right\} \quad\left(\lambda \in \mathbb{C}_{+}\right) .
$$

The function $(1-R V(\lambda))^{-1}$ maps $\mathbb{C}_{+}$into $\mathbb{C}_{+}$as $R>0$, and $-\mathbb{C}_{-}$into $\mathbb{C}_{-}$as $R<0$. For definiteness sake, take $R>0$. Then the argument on the right-hand side of equality (69) belongs to $[0, \pi]$ as $\lambda \in \mathbb{C}_{+}$. This argument is

$$
\arg \frac{\lambda+i}{\lambda-\varkappa}+\int_{\mathbb{R}} \frac{y}{(t-x)^{2}+y^{2}}(\xi(t)-\psi(t)) d t,
$$


where $\lambda=x+i y \in \mathbb{C}_{+}$. Each of the summands in this sum is a harmonic function in $\mathbb{C}_{+}$, in view of the maximum principle, and its maximum and minimum values are achieved on the boundary $\lambda=x+i 0 \in \mathbb{R}$. Taking into account the properties of the Poisson kernel [12], we obtain that the boundary value on $\mathbb{R}$ from $\mathbb{C}_{+}$of $\operatorname{sum}(70)$ is $\pi \psi(x)+\pi(\xi(x)-\psi(x))=\pi \xi(x)$. And thus $0 \leq \xi(x) \leq 1$ for all $x \in \mathbb{R}$ when $R>0$.

Theorem 19. Let $\operatorname{dim} E=1, \sigma_{0}$ and $\sigma_{p}$ (44) be given by $\sigma_{0}=[a, b]$ $(-\infty<a<b<\infty)$ and $\sigma_{p}=\{\varkappa \in \mathbb{R} \backslash[a, b]\}$, and the scattering matrix $S(x)=\exp \{-2 \pi \xi(x)\}$, where $\xi(x)$ is the function of spectral shift (39).

Then $V(\lambda)(2)$ is given by

$$
V(\lambda)=R^{-1}\left\{1-\frac{\lambda-\varkappa}{\lambda+i} \exp \left\{\int_{\mathbb{R}} \frac{\psi(x)-\xi(x)}{x-\lambda} d x\right\}\right\} \quad\left(\lambda \in \mathbb{C}_{+}\right),
$$

where

$$
0 \leq \xi(x) \leq 1 \quad(R>0) \quad(-1 \leq \xi(x) \leq 0, R<0),
$$

the function $\varphi(x)$ is given by $(68)$, and $R^{-1}=V(\varkappa)$.

It should be noticed that in (66) we could choose $i a(a>0)$ instead of $i$. Either for $i a$ or $i$ the final result is the same: formula (71) does not depend on $a$.

By the scattering data, the scattering matrix $S(x)=\exp \{-2 \pi i \xi(x)\}$, and the number $\varkappa \in \sigma_{p}$, using (71), we construct the Nevanlinna function $V(\lambda)$ where the constant $R=V(r)$. Next, knowing $V(\lambda)$, we find its representing measure $d F(x)=a(x) d x$, using which we define the space $L_{\mathbb{R}}^{2}(E, d F(x))(18)$ and the pair of operators $\{\mathcal{Q}, \mathcal{L}\}(20)$. Thus, the Friedrichs-Faddeev model is unambiguously defined by the scattering data $\{S(x), \varkappa\}(20)$.

X. Let $\operatorname{dim} E=1$ and $\sigma_{0}$ be given by

$$
\sigma_{0}=\left[a, c_{1}\right] \cup\left[c_{2}, c_{3}\right] \cup \ldots \cup\left[c_{m}, b\right]
$$

$\left(-\infty<a<c_{1}<c_{2}<\ldots<c_{m}<b<\infty, m \in \mathbb{N}\right)$. Then the discrete spectrum $\sigma_{p}(44)$ may consist of $m$ points $\left\{\varkappa_{k}\right\}_{1}^{m}$ that are zeroes of the equation $1=R V(\lambda)$ and are in the lacunas of the set $\sigma_{0}(73)$. Besides, there are two possibilities: either $\varkappa_{1} \in(-\infty, a), \varkappa_{2} \in\left(c_{1}, c_{2}\right), \ldots, \varkappa_{m} \in\left(c_{m-1}, c_{m}\right)$ as $R>0$; or $\varkappa_{1} \in\left(c_{1}, c_{2}\right), \varkappa_{2} \in\left(c_{3}, c_{4}\right), \ldots, \varkappa_{m} \in(b, \infty)$, when $R<0$. The roots $\left\{\varkappa_{k}\right\}_{1}^{m}$ in the lacunas of the set $\sigma_{0}(73)$ depend on the behavior of the representing measure $d F(x)=a(x) d x$ of the function $V(\lambda)(2)$ at the extremities of zones, i. e., at the points $a<c_{1}<\ldots<c_{m}<b$, as well as on the value of number $R$. 
Similarly to (66), in this case, we write the Riemann boundary value problem (34) as

$$
S(\lambda)\left(\frac{\lambda+i}{\lambda-i}\right)^{m} \prod_{k=1}^{m} \frac{\lambda-\varkappa_{k}}{\lambda+i}\left(1-R V_{+}(\lambda)\right)^{-1}=\prod_{k=1}^{m} \frac{\lambda-\lambda_{k}}{\lambda-i}\left(1-R V_{-}(\lambda)\right)^{-1}
$$

Using again the considerations of the previous section, we obtain the unique solution for this boundary problem,

$$
(1-R V(\lambda))^{-1}=\prod_{k=1}^{m} \frac{\lambda+i}{\lambda-\varkappa_{k}} \exp \left\{\int_{\mathbb{R}} \frac{\xi(x)-m \varphi(x)}{x-\lambda} d x\right\} \quad\left(\lambda \in \mathbb{C}_{+}\right),
$$

where $\xi(x)$ is the function of spectral shift (39), and $\psi(x)$ is given by (68). In this case, Theorem 19 can be formulated as follows.

Theorem 20. Let $\operatorname{dim} E=1$ and $\sigma_{0}$ be given by (73), and the set $\sigma_{p}$ (44) be $\sigma_{p}=\left\{\varkappa_{k} \in \mathbb{R} \backslash \sigma_{0}, 1 \leq k \leq m\right\}$. Then the function $V(\lambda)$ (2) is defined unambiguously by the collection $\left\{S\left(x,\left\{\varkappa_{k}\right\}_{1}^{m}\right\}\right.$,

$$
V(\lambda)=R^{-1}\left\{1-\prod_{k=1}^{m} \frac{\lambda-\varkappa_{k}}{\lambda+i} \exp \left\{\int_{\mathbb{R}} \frac{m \psi(x)-\xi(x)}{x-\lambda} d x\right\}\right\} \quad\left(\lambda \in \mathbb{C}_{+}\right),
$$

$\xi(x)$ is the function of spectral shift $(39)(S(x)=\exp \{-2 \pi i \xi(x)\})$ and

$$
0 \leq \xi(x) \leq 1 \quad(R>0) ; \quad(-1 \leq \xi(x) \leq 0, R<0),
$$

where $\varphi(x)$ is given by $(68)$, and $R^{-1}=V\left(\varkappa_{n}\right)(1 \leq k \leq m)$.

We can conclude that the Friedrichs-Faddeev model $\{\mathcal{Q}, \mathcal{L}\}(20)$ is defined unambiguously by the scattering matrix $S(x)$ and the collection of numbers $\left\{\varkappa_{k}\right\}_{1}^{m}$.

\section{References}

[1] M. Reed and B. Simon, Methods of modern mathematical physics. III: Scattering Theory. Academic Press, Inc., 1979.

[2] D.R. Yafaev, Mathematical Scattering Theory: General Theory. Transl. of Math. Monographs, 105, AMS, Providence, RI, 1992.

[3] H. Baumgärtel and M. Wollenberg, Mathematical Scattering Theory. Math. Textbooks and Monogr., vol. 59, Part II; Mathem. Monogr., Akademie-Verlag, Berlin, 1983.

[4] V.A. Marchenko, Sturm-Liouville Operators and Applications. AMS Chelsea Publishing, Revised edition, 2011. 
[5] L.D. Faddeev, The Inverse Problem in the Quantum Theory of Scattering. - J. Math. Phys. 4 (1963), 72-104.

[6] C. Shadan and P. Sabatier, Inverse Problems in Quantum Scattering Theory, Springer, 1989.

[7] B.M. Levitan, Inverse Sturm Liouville Problems. VMU Science Press, Utrecht, 1987.

[8] J.F. Brasche, M.M. Malamud, and H. Neidhardt, Weyl Function and Spectral Properties of Self-Adjoint Extensions. - Integr. Eqs., Oper. Theory 43 (2002), No. 3, 264-289.

[9] J.F. Brasche, M.M. Malamud, and H. Neidhardt, Scattering Theory for Open Quantum Systems with Finite Rang Coupling. - Math. Phys., Anal., Geom. 10 (2007), 331-358.

[10] J.F. Brasche, M.M. Malamud, and H. Neidhardt, Scattering Matrices and Weyl Functions. - Proc. London Math. Soc. 97 (2008), No. 3, 568-598.

[11] R. Tiedra de Aldecoa, Time Delay for Dispersive Systems in Quantum Scattering Theory. - Rev. Math. Phys. 21 (2009), No. 5, 675-708.

[12] N.I. Akhiezer, The Classical Moment Problem and Some Related Questions in Analysis. Oliver \& Boyd, 1965.

[13] N.I. Akhiezer and I.M. Glazman, Theory of Linear Operators in Hilbert Space, vol. 2, 3rd ed. Boston, Mass. - London, Pitman (Advanced Publishing Program), 1981.

[14] J.B. Garnett, Bounded Analytic Functions (Graduate Texts in Mathematics). Springer, New York, 2006.

[15] P. Koosis, Introduction to $H^{p}$ Spaces. Cambridge University Press, Vol. 40, Cambridge, 1980.

[16] F.D. Gahov, Boundary Problems. M. Fiz.-mat. lit., 1977. (Russian)

[17] I.C. Gohberg and M.G. Krein, Introduction to the Theory of Linear Nonselfadjoint Operators in Hilbert Space. Vol. 18. AMS, 1969.

[18] M.G. Krein, On the Trace Formula in Perturbation Theory. - Mat. Sb. (N.S.), 33(75):3 (1953), 597-626. (Russian)

[19] M. Anthea Grubb and D.B. Pearson, Derivation of the Wave and Scattering Operators for Interactions of Rank One. - J. Math. Phis. 11 (1970), 2415-2424.

[20] J. Kellendonk and S. Richard, On the Structure of the Wave Operators in OneDimensional Potential Scattering. - Math. Phys. Electron. J. 14 (2008), 13-21.

[21] S. Richard and R. Tiedra de Aldecoa, New Formulae for the Wave Operators for a Rank One Interaction. - Integr. Equation, Oper. Theory 66 (2010), No. 2, 283-292. 